\title{
Dual-Ligand-Modified Liposomes Co-Loaded with Anti-Angiogenic and Chemotherapeutic Drugs for Inhibiting Tumor Angiogenesis and Metastasis
}

\author{
Fangqing Wang ${ }^{1, *}$ \\ Yanying $\mathrm{Li}^{1, *}$ \\ Hong Jiang $\mathbb{D}^{1, *}$ \\ Chenglei $\mathrm{Li}^{2}$ \\ Zhaohuan $\mathrm{Li}^{2}$ \\ Cuiping $\mathrm{Qi}^{3}$ \\ Zhipeng $\mathrm{Li} \mathbb{1}^{\prime}$ \\ Zhiqin Gao' \\ Bo Zhang ${ }^{2}$ \\ Jingliang Wu (D)
}

'School of Bioscience and Technology, Weifang Medical University, Weifang,

26I053, People's Republic of China;

${ }^{2}$ School of Pharmacy, Weifang Medical University, Weifang, 261053, People's

Republic of China; ${ }^{3}$ School of Nursing, Weifang Medical University, Weifang,

261053, People's Republic of China

*These authors contributed equally to this work

\begin{abstract}
Background: Tumor angiogenesis has been proven to potentiate tumor growth and metastasis; therefore, the strategies targeting tumor-related angiogenesis have great potentials in antitumor therapy.

Methods: Here, the GA\&Gal dual-ligand-modified liposomes co-loaded with curcumin and combretastatin A-4 phosphate (CUCA/GA\&Gal-Lip) were prepared and characterized. A novel "BEL-7402+HUVEC" co-cultured cell model was established to mimic tumor microenvironment. The cytotoxicity and migration assays were performed against the novel co-cultured model. Angiogenesis ability was evaluated by tube formation test, and in vivo metastatic ability was evaluated by lung metastasis test.

Results: The result demonstrated that dual-ligand-modified liposomes showed greater inhibition of tumor angiogenesis and metastasis in comparison with other combined groups. Significantly, the mechanism analysis revealed that curcumin and combretastatin A-4 phosphate could inhibit tumor angiogenesis and metastasis via down-regulation of VEGF and VEGFR2 expression, respectively, and that GA\&Gal-Lip could improve antitumor effect by GA/Gal-mediated active-targeting delivery.
\end{abstract}

Conclusion: CUCA/GA\&Gal-Lip hold great potentials in hepatoma-targeting delivery of antitumor drugs and can achieve anti-angiogenic and anti-metastatic effects by simultaneously blocking VEGF/VEGFR2 signal pathway, therefore exhibiting superior antihepatoma efficacy.

Keywords: dual-ligand-modified, liposomes, anti-angiogenesis, VEGF, co-delivery

\section{Introduction}

Liver cancer is one of the most common cancers worldwide with high morbidity and mortality. ${ }^{1,2}$ Chemotherapy is still the fundamental anti-hepatoma method in clinic, while the single treatment cannot effectively inhibit tumor development and metastasis due to severe systemic toxicity and drug resistance. ${ }^{3}$ Combination therapy is acknowledged to be an effective strategy owing to superior antitumor efficacy by the synergistic effect of different drugs. ${ }^{4,5}$ In recent years, antiangiogenic therapy has been widely accepted as a promising antitumor strategy to block the development of tumor angiogenesis. ${ }^{6}$ Tumor growth and metastasis depend on rich blood vessels to provide nutrition. The activation and proliferation of vascular endothelial cells is an important factor in promoting angiogenesis. Many studies have shown that the vascular endothelial growth factor (VEGF) is one of the most effective vascular growth factors. ${ }^{7}$ VEGF promotes angiogenesis by specifically binding to vascular endothelial growth factor
Correspondence: Bo Zhang; Jingliang Wu Email zhangbo@wfmc.edu.cn;

jlwu2008@I63.com 
receptor 2 (VEGFR2). The VEGF can bind to VEGFR2 that leads to the phosphorylation of VEGFR2 and activate the downstream pathway of VEGFR2, which can promote migration and proliferation of vascular endothelial cells (Figure 1A). Therefore, blocking the VEGF/VEGFR2 signaling pathway is considered to be an effective strategy to inhibit tumor angiogenesis and tumor growth (Figure 1B). The combination of chemotherapeutics with antiangiogenic drugs is expected to improve therapeutic efficacy for HCC. ${ }^{8,9}$

Combretastatin A-4 phosphate (CA4P), a vascular disrupting agent (VDA) undergoing clinical research, could inhibit proliferation and migration of vascular endothelial cells by blocking VEGF-induced autophosphorylation of VEGFR2. ${ }^{10}$ On the other hand, curcumin (CUR) has been shown to have effects on the pro-apoptotic activity in various cancer cells by inhibiting the activation of PI3-kinase /AKT, JAK/STAT3 and NF-кB. ${ }^{11-14}$ Meanwhile, CUR also could inhibit tumor angiogenesis by down-regulating VEGF expression of tumor cells. ${ }^{15,16}$ In consideration of this, the combination of CUR and CA4P may significantly inhibit tumor angiogenesis by blocking VEGF/VEGFR2 signaling pathway, leading to efficient antitumor effects. However, both free drugs possess undesired systemic toxicity, low bioavailability and lack of antitumor selectivity. Moreover, due to different physicochemical properties between CUR and CA4P, it is difficult to exert their synergistic effects for conventional combinational therapy. Therefore, it is urgent to design an efficient and safe drug delivery system for co-delivery of CUR and CA4P.

Liposomes (Lip) have been considered the effective nano-sized carriers for combination therapy due to their inherent ability to simultaneously encapsulate both hydrophilic and hydrophobic drugs. ${ }^{17}$ In order to improve drug accumulation and cellular uptake in tumor region, the Lip could be modified by different ligands, such as antibody, sugars and other moieties. ${ }^{18}$ Compared to normal liver tissue, the protein kinase $\mathrm{C} \alpha$ and asialoglycoproteinreceptor (ASGP-R) are over-expressed on the surface of HCC cells, so the ligands, which could bind to these proteins, are introduced to the shell of Lip to improve hepatoma-targeting delivery. Recent researches confirmed that glycyrrhetinic acid (GA) and galactose (Gal) can specifically bind the protein kinase $\mathrm{C} \alpha$ and asialoglycoproteinreceptor, respectively. ${ }^{19-21}$ However, single ligand often caused off-target effects due to insufficient responsiveness. In addition, Lip modified by only a single ligand might not effectively be taken up by tumor cells when the receptors were saturated by other nano-carriers. ${ }^{22-24}$ Consequently, the dual-ligand-modified Lip can be designed to promote cellular uptake, and improve antitumor efficacy.

Numerous evidences have verified that tumor microenvironment (TME) plays a crucial role in tumor development. $^{25}$ In the TME, the stromal cells, such as fibroblasts, endothelial cells and immune-related cells, are associated with tumor growth, invasion and metastasis. ${ }^{26-29}$ Regrettably, most of the antitumor evaluation models were currently processed only in tumor cells alone, which cannot reflect the real complexity in the tumor region. This might be why certain drugs finally failed in clinic, although exhibited good effectiveness in preclinical studies. In the present work, on the basis of single tumor cells model, we also proposed a co-cultured system composed of tumor cells and vascular endothelial cells to further investigate the anti-angiogenic and antimetastatic effects, which would be more effective to evaluate the actual antitumor effects of the formulations.

Summarily, the co-loaded Lip (CUCA/GA\&Gal-Lip) modified by GA and Gal were prepared in this study, which aimed to explore the inhibition of tumor angiogenesis and metastasis by down-regulating VEGF and VEGFR2 expression simultaneously. Two-level evaluation models were established to evaluate the cytotoxicity and migration of coloaded Lip against HCC cells alone and HUVEC/HCC cocultured cells. Additionally, anti-hepatoma activity and lung metastasis assay in vivo were performed to investigate the efficacy of anti-angiogenesis, anti-proliferation and inhibition of HCC metastasis. For better understanding, this is the first attempt for GA\&Gal dual-ligand-modified Lip co-loaded with anti-angiogenic and chemotherapeutic drugs to be systematically evaluated in HUVEC/HCC co-cultured model.

\section{Materials and Methods Materials}

Egg phosphatidylcholine (EPC) and cholesterol were purchased from A.V.T (Shanghai, China). CUR and MTT were purchased from Solarbio (Beijing, China). CA4P were obtained from Aladdin Chemistry Co. Ltd. (China). Matrigel matrix was purchased from BD Biosciences (USA). DAPI was obtained from Sigma-Aldrich (USA). DSPE (1,2-distearoyl-sn-glycero-3-phosphoethanolamine)PEG (polyethylene glycol)-FITC (fluorescein isothiocyanate) and DSPE-PEG-NHS (N-hydroxysuccinimide) were obtained from Xi'an Ruixi Biological Technology Co., Ltd. (China). DSPE-PEG-Gal and DSPE-PEG-GA were synthesized in laboratories. DiR (near-infrared membrane probe) 
A

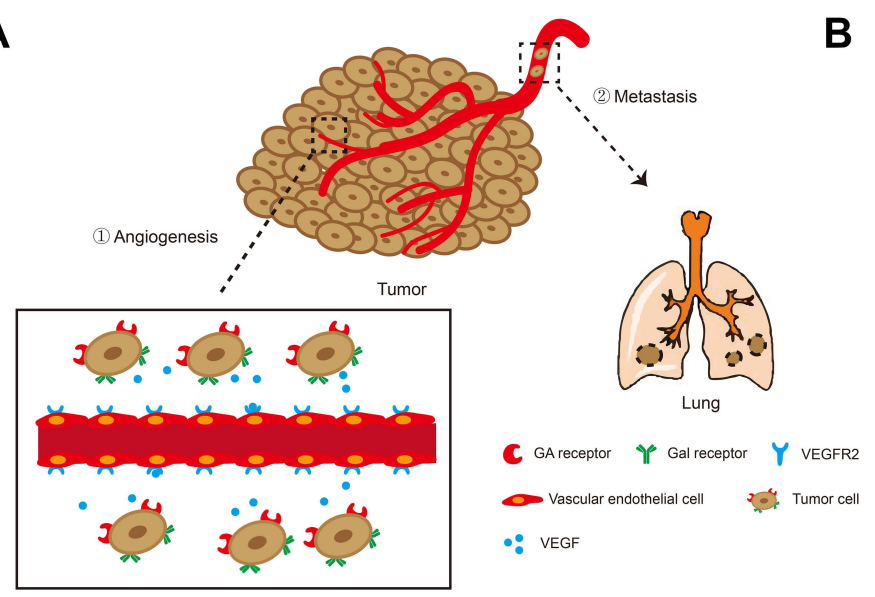

B

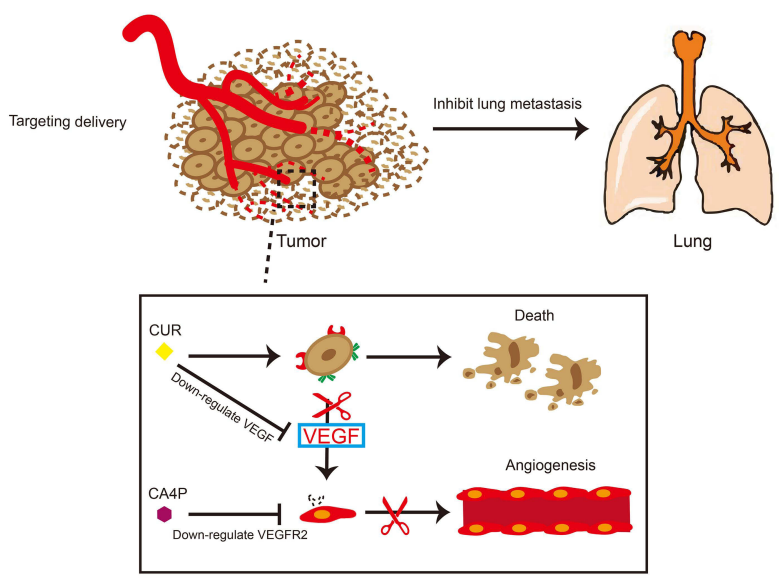

C
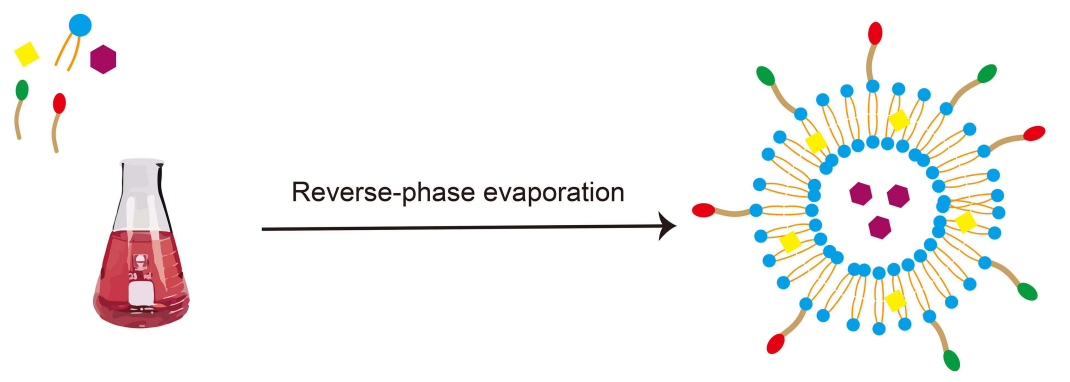

Phospholipid

CA4P

CUR

DSPE-PEG-GA

DSPE-PEG-Gal

CUCA/GA\&Gal-Lip

Figure I (A) Schematic illustration of angiogenesis and lung metastasis. (B) Schematic illustration of antitumor mechanism and inhibit lung metastasis. (C) Illustration of liposomal preparations.

was purchased from Beijing Innochem Co., Ltd. (China). Anti-CD31 antibody was purchased from Abcam (USA). All other chemicals and reagents in this study were of analytical grade.

\section{Cell Lines and Animals}

Human hepatocellular carcinoma cell line (BEL7402 cells), murine HCC cells (H22) and human umbilical vein endothelial cells (HUVEC) were obtained from Biotechnology Research Institute (Beijing, China). BEL7402 and HUVEC cells were cultured in RPMI 1640 and HUVEC special medium, respectively. H22 cells were injected subcutaneously into $\mathrm{BALB} / \mathrm{c}$ mice to build hepatoma-bearing mice model. BALB/c mice (female,18 22g) were obtained from Jinan Pengyue Lab animal center (China). All animal experiments were approved by the Animal Experiment Ethics Committees of WeiFang Medical University (WFMC, 2019-045), and followed the welfare and treatment of the laboratory animals strictly complied with the Animal Management Rules of the
Ministry of Health of the People's Republic of China (document no. 55, 2001).

\section{Preparation of Co-Loaded Lip Modified by GA\&Gal}

DSPE-PEG-GA was synthesized by a two-step reaction. ${ }^{30}$ In brief, GA was activated by DMTMM (cross-linking agent), followed by dropping ethylene diamine to obtain diamine-modified GA (GA-N). DSPE-PEG-GA was synthesized by adding GA-N to DSPE-PEG-NHS solution at a molar ratio of $3: 1$ in the presence of EDC. DSPE-PEG -Gal was synthesized by the reaction of DSPE-PEG$\mathrm{COOH}$ and D-GalN in the presence of EDC and NHS in our previous study. ${ }^{31}$ CUCA/GA\&Gal-Lip were prepared by reverse-phase evaporation method (Figure 1C). Firstly, EPC and cholesterol were mixed in chloroform (ratio of EPC and cholesterol was 3:1), then DSPE-PEG-Gal, DSPE-PEG-GA and Cur were added to this system (the ratios of DSPE-PEG-GAL/EPC, DSPE-PEG-GA/EPC and Cur/EPC were 1:20). Secondly, $15 \mathrm{mg}$ of CA4P was 
dissolved in $3 \mathrm{~mL}$ of PBS, and transferred to the lipid solution. The mixed system was sonicated for 5 minutes to produce a water-in-oil (W/O) uniform emulsion. Chloroform was removed under decompression rotating evaporation to form the gel. Next, $3 \mathrm{~mL}$ PBS was added and hydrated at $60{ }^{\circ} \mathrm{C}$ for $1 \mathrm{~h}$. Finally, the Lip were sonicated for $7 \mathrm{~min}$ in an ice bath and passing sequentially the $0.45-\mu \mathrm{m}$ and $0.22-\mu \mathrm{m}$ syringe filters for 3 times.

\section{Characterization of CUCA/GA\&Gal-Lip}

The average particle size and zeta potential of CUCA/ GA\&Gal-Lip were measured by a Malvern Zetasizer Nano ZS90. All samples were diluted 10-fold with PBS before analysis. To evaluate the colloidal stability, CUCA/ GA\&Gal-Lip were dispersed in PBS or RPMI 1640 at $4^{\circ}$ $\mathrm{C}$ for 14 days, and the particles size was measured every 2 days. The morphologies of CUCA/Lip and CUCA/ GA\&Gal-Lip were observed by transmission electron microscope (TEM). The loading efficiency (LE) and encapsulation efficiency (EE) were important evaluation indexes for nano-sized drug delivery system. In this study, the content of CUR was detected at the absorption wavelength of $425 \mathrm{~nm}$ using an ultraviolet (UV) spectrophotometer, while CA4P was determined by high-performance liquid chromatography (HPLC) at 295nm. LE and EE of were calculated by the following equations:

$$
\begin{aligned}
& L E=\frac{\text { weight of encapsulated drug }}{\text { weight of all materials }} \times 100 \% \\
& E E=\frac{\text { weight of encapsulated drug }}{\text { weight of initial added drug }} \times 100 \%
\end{aligned}
$$

\section{In vitro Drug Release from Lip}

To investigate the drug release profiles from Lip, CUCA/ GA\&Gal-Lip was transferred to the dialysis bag (MW cut-off $3500 \mathrm{Da}$ ) and then immersed into $50 \mathrm{~mL}$ of PBS (containing $1 \%$ Tween 80 ) at $\mathrm{pH}=7.4 .^{30}$ The release system was continuously kept at a speed of $100 \mathrm{rpm}$ at $37{ }^{\circ} \mathrm{C}$ in a shaking incubator (THZ-82, Jintan, China). At 0.5, 1, 2, 4, 8, 12, 24, 36 and $48 \mathrm{~h}, 4 \mathrm{~mL}$ of release medium were removed and equal volume of PBS medium were supplied. The content of CUR was determined by UV at $425 \mathrm{~nm}$ and CA4P was detected by HPLC at $295 \mathrm{~nm}$. Cumulative release percentage was calculated according to the following formula:

$$
E r=\frac{V_{r} \sum_{1}^{n-1} C_{i}+V_{0} C_{n}}{M_{d}} \times 100 \%
$$

$M_{d}$ is the content of drugs in the dialysis bag. $V_{0}$ is the initial volume of PBS medium. $V_{r}$ is the volume of the release medium per take out, and $C_{i}$ is the concentration of CUR or CA4P in the release medium.

\section{Cellular Uptake}

BEL7402 cells were seeded in the cell culture dishes (35$\mathrm{mm}$ glass bottom petri dish) at $5 \times 10^{4}$ cells/dish and cultured overnight at $37^{\circ} \mathrm{C}$. Then, the cells were co-incubated with Fluorescein isothiocyanate (FITC)-labeled Lip, FITClabeled Gal-Lip or FITC-labeled GA\&Gal-Lip for 0.5 $\mathrm{h}$ and washed thrice with PBS. The BEL7402 cells were fixed with 4\% paraformaldehyde for 10-15 $\mathrm{min}$, and nuclei were stained with DAPI for $10 \mathrm{~min}$. Subsequently, the cellular uptake of different treatment groups was observed via confocal laser scanning microscopy. All measurements were performed in triplicate.

\section{In vitro Anti-Proliferate Effect}

The anticancer effects of different treatment groups were investigated by MTT assay as previously described. ${ }^{32}$ BEL7402 cells alone and BEL7402/HUVEC co-cultured cells (ratio of BEL7402 to HUVEC cells was 5:1) were seeded in 96-well plates at a density of $6 \times 10^{3}$ cells/well and then cultured for 24 hours at $37^{\circ} \mathrm{C}$, respectively. After the medium was replaced with free CUR solution, free CA4P solution, mixture of CUR and CA4P, CUCA/Lip, CUCA/Gal-Lip, CUCA/GA\&Gal-Lip, respectively, these cells were incubated for $48 \mathrm{~h}$. Subsequently, $10 \mu \mathrm{L}$ of MTT $(5 \mathrm{mg} / \mathrm{mL})$ was added to the 96-well plate for further incubation. Then, the media in each well were thrown away, followed by addition of $150 \mu \mathrm{L}$ of DMSO. The absorbance of DMSO solution was measured at the wavelength of $490 \mathrm{~nm}$ using a microplate reader (ELX800, BioTek, USA). All measurements were performed in triplicate.

\section{Live/Dead Staining}

The Calcein-AM/PI double-staining kit from Meilun (Dalian, China) was used to measure cell viability. The cells were double-stained with two probes (Calcein-AM for live cells, PI for dead cells). ${ }^{33}$ Briefly, BEL7402 cells $\left(6 \times 10^{3}\right.$ cell/well $)$ or co-cultured system of BEL7402 and HUVEC cells (ratio of 5 to 1 ) were treated with different drug formulations for $48 \mathrm{~h}$. After 48 h, $2 \mu \mathrm{M}$ Calcein-AM and $8 \mu \mathrm{M}$ PI were added to the wells. Then, the cells were cultured for another $30 \mathrm{~min}$ in the dark. Images were acquired using a Nikon eclipse Ti-S microscope. 


\section{Tube Formation Assays}

The inhibitory effect of drug-loaded Lip on the angiogenesis was evaluated using the tube forming assay. ${ }^{34}$ Briefly, $50 \mu \mathrm{L}$ matrigel were added to 96 -well plates, followed by incubation for $30 \mathrm{~min}$. Then, $90 \mu \mathrm{L}$ of HUVEC cells' suspension (about $1.1 \times 10^{4}$ cells) was seeded in 96-well plates, and $10 \mu \mathrm{L}$ of PBS, free CUR, free CA4P, mixture of two free drugs, CUCA/Lip, CUCA/Gal-Lip, or CUCA/ GA\&Gal-Lip were added, respectively. After incubation for another $6 \mathrm{~h}$, the representative images were photographed using a Nikon eclipse Ti-S microscope. The HUVEC cell tube formation was quantitatively measured using Image $\mathbf{J}$ software.

\section{Cell Migration Assays}

HUVEC cells were harvested and washed 3 times with PBS. Then, the cells were resuspended with $5 \mu \mathrm{M}$ Carboxyfluorescein succinimidyl ester (CFSE) and incubated for $30 \mathrm{~min}$ at $37{ }^{\circ} \mathrm{C}$. The reaction was terminated for 1 min by FBS, and the CFSE-labeled HUVEC cells were washed 2 times with PBS. ${ }^{35}$ BEL7402 cells alone and BEL7402/HUVEC co-cultured cells (ratio of BEL7402 to HUVEC cells was 5:1) were seeded in 6-well plates at a density of $6 \times 10^{5}$ cells/well with incubation for $24 \mathrm{~h}$ at 37 ${ }^{\circ} \mathrm{C}$, respectively. Then three lines were scratched with 200 $\mu \mathrm{L}$ pipette tips in each well and washed thrice with PBS Subsequently, different drug formations were added to different wells for another $24 \mathrm{~h}$. At both 0 and $24 \mathrm{~h}$, the wounds width was captured using a Nikon eclipse Ti-S microscope. The anti-migration effect of treatment groups was evaluated by the percent of gap closure, which was calculated according to the formula:

$$
\text { Migration rate }=\left(1-\frac{\text { Width }_{0 h}-\text { Width }_{24 h}}{\text { Width }_{0 \mathrm{~h}}}\right) \times 100 \%
$$

\section{Immunofluorescence Analysis of VEGF Expression}

BEL 7402 cells were seeded in cell culture dishes at $5 \times 10^{4}$ cells/dish and cultured for $24 \mathrm{~h}$ at $37^{\circ} \mathrm{C}$. After incubation with different drug formulations for another $24 \mathrm{~h}$, the cells were then washed thrice with PBS, and fixed with $4 \%$ paraformaldehyde for $15 \mathrm{~min}$. All the sample wells were incubated with the primary antibody of VEGF overnight at $4{ }^{\circ} \mathrm{C}$, followed by incubation with the secondary antibody for $1.5 \mathrm{~h}$ at room temperature. The cells were stained with DAPI for 8 min before imaging.

\section{In vivo Imaging Analysis}

To observe the in vivo biodistribution of dual-ligandmodified Lip, hepatoma-bearing mice model was established by subcutaneous injection $0.1 \mathrm{~mL}$ of $\mathrm{H} 22$ cells suspension into the BALB/c mice. ${ }^{36}$ When the tumor volume reached about $150 \mathrm{~mm}^{3}$, the mice were divided into three groups and intravenously injected with free DiR, DiR/Lip or DiR/GA\&Gal-Lip. The DIR biodistribution in mice was investigated using in vivo imaging system at 1 , $2,4,8,12,24,48$ and $72 \mathrm{~h}$ after injection. After $72 \mathrm{~h}$, the mice were euthanatized, and the important organs and tumor were taken out for further analysis of radiation intensity.

\section{In vivo Antitumor Efficacy}

$\mathrm{H} 22$ hepatoma-bearing BALB/c mice model were established to investigate the antitumor effects of the CUCA/ GA\&Gal-Lip in vivo. In this study, 40 female BALB/c mice were used for the antitumor study in vivo. When the tumor grew to approximately $100 \mathrm{~mm}^{3}$, the mice were randomly divided into 7 treatment groups $(\mathrm{n}=4$ for each group). Then, the mice of different groups were injected with free CUR, free CA4P, mixture of CUR and CA4P, CUCA/Lip, CUCA/Gal-Lip, and CUCA/GA\&Gal-Lip via tail vein, respectively. The drug dosage of each group was applied at at equivalent dosage of CUR $(5 \mathrm{mg} / \mathrm{kg})$ and/or that of CA4P every 2 days. The mouse weights and tumor volumes were measured every 2 days. Two weeks later, the mice were sacrificed, and the important organs and tumor were harvested for further study. The tumor inhibition rate was calculated:

$$
\left(1-\frac{\text { tumor weight of drug }- \text { treated group }}{\text { tumor weight of control group }}\right) \times 100 \%
$$

\section{Lung Metastasis Model and Treatments}

$\mathrm{H} 22$ cells $\left(1 \times 10^{7}\right.$ cells $\left./ \mathrm{mL}\right)$ were intravenously injected into the female $\mathrm{BALB} / \mathrm{c}$ mice to allow the formation of lung metastasis. ${ }^{37,38}$ Then, the mice were randomly divided into seven groups ( $\mathrm{n}=4$ per group) and intravenously injected with saline, free CUR, free CA4P, mixture of two free drugs, CUCA/Lip, CUCA/Gal-Lip, CUCA/ GA\&Gal-Lip (equivalent dosage of CUR $(5 \mathrm{mg} / \mathrm{kg})$ and $/$ or that of CA4P every 2 days) for 14 days. After 14 days, all mice were sacrificed, and the number of metastatic lung nodules was measured. 


\section{Histochemical Staining and Immunohistochemistry Analysis}

The samples of tumor and different organs were fixed with $4 \%$ paraformaldehyde. These tissues were embedded in paraffin blocks and cut into sections. Subsequently, hematoxylin and eosin (H\&E) were used to stain these sections. CD31, VEGF and VEGFR2 antibody were used to detect tumor microvascular proliferation. ${ }^{39}$ All the samples were observed using fluorescence microscopy (OlympusIX51, Japan).

\section{Statistical Analysis}

Statistical analysis was performed using Prism 8.0 (GraphPad). All experimental data were shown by mean \pm standard deviation (SD). Differences between the treatment groups were determined by Student's $t$ test, and $\mathrm{P}<0.05$ was considered as statistical significance.

\section{Results and Discussion}

\section{Preparation and Characteristics of}

\section{CUCA/GA\&Gal-Lip}

Dual-ligand-modified Lip for co-delivery of CUR and CA4P were prepared, and the average particles sizes, PDI, $\zeta$ potentials, EE and LE of Lip are presented in Table 1. The average particle sizes of three liposomal formulations were around $150 \mathrm{~nm}$ and PDI was about 0.15 . The $\zeta$-potentials were negatively. The EE and LE of CUR in CUCA/ GA\&Gal-Lip were $83.3 \%$ and $2.72 \%$, respectively, while the EE and LE of CA4P in CUCA/GA\&Gal-Lip were $43.8 \%$ and $3.58 \%$, respectively. The EE of CA4P was only $43.8 \%$, which might be related to the water-solubility of CA4P. Additionally, the TEM images revealed that drugloaded Lip were spherical in shape (Figure 2A-C). Figure $2 \mathrm{D}$ shows that there was no significant change in particle size of CUCA/GA\&Gal-Lip in PBS or RPMI 1640 at $4{ }^{\circ} \mathrm{C}$ for 14 days. This result indicated that the drug-loaded Lip were highly stable. Furthermore, in vitro drug release profiles from CUCA/GA\&Gal-Lip are shown in Figure 2E. Compared to CUR, CA4P exhibited burst release with around $50 \%$ of CA4P was released in the first 4 hours. After $8 \mathrm{~h}$, the release rate of both drugs decreased, and the sustained release was kept within $48 \mathrm{~h}$. In $48 \mathrm{~h}, 73.4 \%$ of CA4P was released from CUCA/GA\&Gal-Lip, whereas $39.3 \%$ of CUR was released. This is due to the fact that CA4P was water-soluble, and escaped from Lip more easily than hydrophobic CUR.

\section{Cellular Uptake Analysis}

To determine the cellular uptake of Lip, BEL7402 were incubated with different FITC-labeled liposomal formulations for $0.5 \mathrm{~h}$. FITC (green fluorescence) was used to track the cellular distribution of Lip, and DAPI (blue fluorescence) was used to stain the nuclei. As shown in Figure 3A and B, the tumor cells treated with non-targeted Lip indicated weak green fluorescence. In contrast, the cells co-incubated with Gal-Lip or GA\&Gal-Lip showed stronger green fluorescence, suggesting that the introduction of Gal or Gal ligand on Lip could promote the cellular uptake. Compared to GalLip, as expected, GA\&Gal-Lip, which were modified by GA and Gal ligand simultaneously, exhibited more green signals in the cytoplasm. This might be due to the fact that the proteins of kinase $\mathrm{C} \alpha$ (GA receptor) and ASGP-R are overexpressed on the surface of $\mathrm{HCC}$ cells, and the dual-ligandmodified Lip could promote cellular endocytosis mediated by both receptors. ${ }^{40,41}$ Overall, GA\&Gal-modified Lip could be taken up by HCC cells more effectively, which would be significant to deliver anti-hepatoma drugs into the cells.

\section{Inhibitive Effect on HUVEC Tube}

\section{Formation in vitro}

To evaluate the anti-angiogenic potentials of combination therapy, tube formation assay was performed. As shown in Figure $4 \mathrm{~A}$ and $\mathrm{B}$, compared to free CUR, free CA4P exhibited stronger ability to inhibit tube formation. Interestingly, there were fewer tube-like structures in the liposomal groups in comparison with mixture of CUR and CA4P, suggesting that co-loaded Lip had stronger antiangiogenic effects. The results showed that CUR and CA4P could be delivered efficiently to endothelial cells using liposomal formulation. Moreover, there was not obvious difference in tube formation rate between the CUCA/Lip and CUCA/Gal-Lip, suggesting that the expression of Gal receptor was almost absent in vascular endothelial cells compared with liver cancer cells.

\section{In vitro Cytotoxicity Assays}

Tumor angiogenesis promotes HCC development and metastasis. To investigate the in vitro cytotoxicity of different drug formulations, both MTT and Live/Dead assay were carried out on two-level cell culture systems (Figure 4C, Supporting Figure 1): BEL7402 cells alone or BEL7402/HUVEC cocultured cells. As shown in Figure 4D and E, all drug treatment groups showed concentration-dependent cytotoxicity against BEL7402 cells and co-cultured system. Meanwhile, 
Table I Characterizations of Various Liposomal Formulations

\begin{tabular}{|c|c|c|c|c|c|}
\hline & DLS (nm) & PDI & $\zeta$-Potential (mV) & EE (\%) & LE (\%) \\
\hline CUCA/Lip & $147.2 \pm 4.3$ & $0.18 \pm 0.02$ & $-2.9 \pm 0.3$ & $\begin{array}{l}87.5 \pm 0.8 \text { (CUR) } \\
47.3 \pm 2.1 \text { (CA4P) }\end{array}$ & $\begin{array}{l}3.05 \pm 0.03 \text { (CUR) } \\
4.11 \pm 0.18 \text { (CA4P) }\end{array}$ \\
\hline CUCA/Gal-Lip & $152 \pm 5.1$ & $0.15 \pm 0.02$ & $-3.1 \pm 0.6$ & $\begin{array}{l}85.5 \pm 1.0 \text { (CUR) } \\
45.4 \pm 0.8 \text { (CA4P) }\end{array}$ & $\begin{array}{l}2.88 \pm 0.03 \text { (CUR) } \\
3.82 \pm 0.06 \text { (CA4P) }\end{array}$ \\
\hline CUCA/GA\&Gal-Lip & $154.9 \pm 4.9$ & $0.18 \pm 0.01$ & $-3.2 \pm 0.1$ & $\begin{array}{l}83.3 \pm 1.3 \text { (CUR) } \\
43.8 \pm 1.3 \text { (CA4P) }\end{array}$ & $\begin{array}{l}2.72 \pm 0.04 \text { (CUR) } \\
3.58 \pm 0.10 \text { (CA4P) }\end{array}$ \\
\hline
\end{tabular}

the CUCA/GA\&Gal-Lip exhibited higher toxicity than CUCA/Lip and CUCA/Gal-Lip. It might be attributed to the stronger liver-targeting capability of dual-ligandmodified Lip, which was shown in the cellular uptake analysis (Figure 3). Interestingly, the $\mathrm{IC}_{50}$ of free CUR and free CA4P against co-cultured system were 5.93 and $12.98 \mu \mathrm{g} /$ $\mathrm{mL}$, respectively, which were 1.8 -fold and 1.7 -fold higher than that of free CUR $(3.17 \mu \mathrm{g} / \mathrm{mL})$ and free CA4P $(7.61 \mu \mathrm{g} /$
$\mathrm{mL}$ ) against BEL7402 cells alone, indicating that the cytotoxicity of single CUR or CA4P was weakened in the presence of BEL7402 and HUVEC cells simultaneously. The possible explanation was that BEL 7402 cells could promote HUVEC proliferation by secreting VEGF factors, and the interactions between BEL7402 and HUVEC cells decreased the antitumor effects of single CUR or CA4P in the cocultured system. As expected, the combination therapy
A

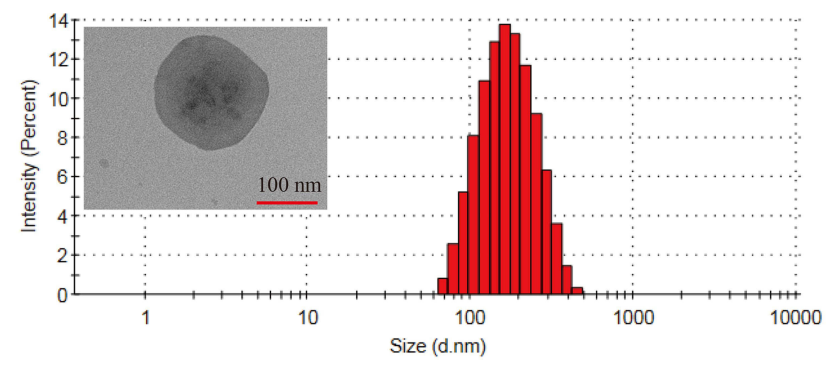

B

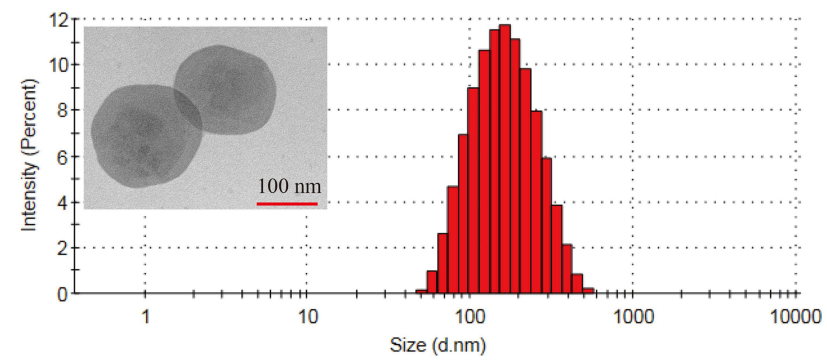

C

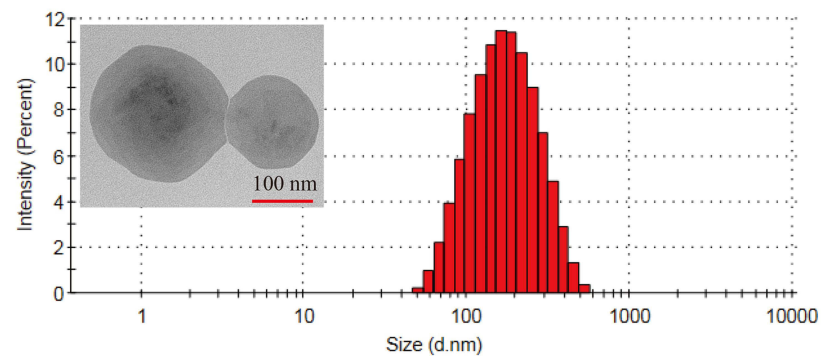

D

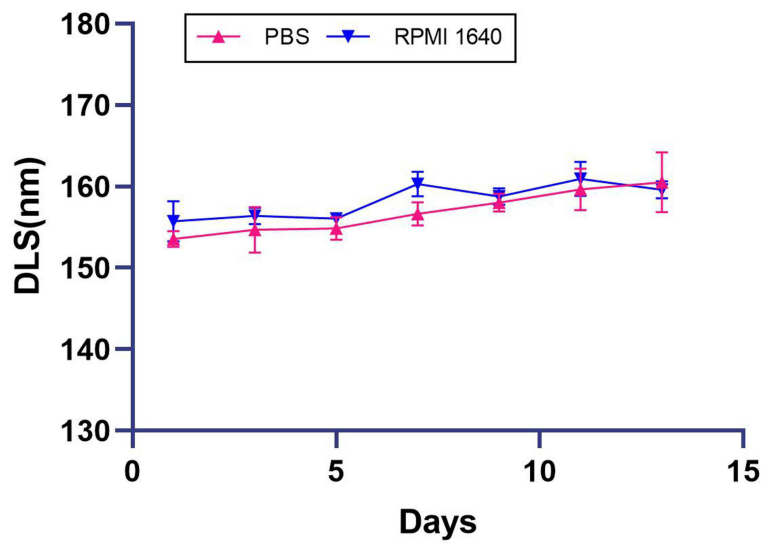

E

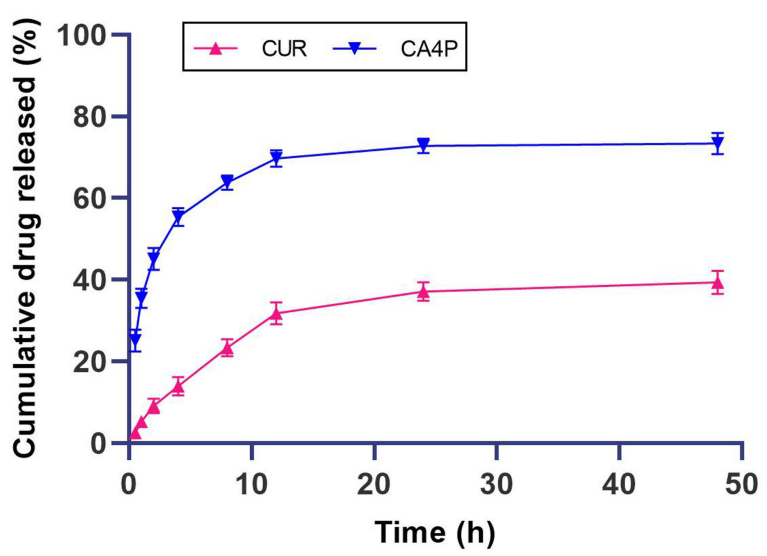

Figure 2 Characterizations of drug-loaded Lip. Particle-size distribution and representative TEM photographs of (A) CUCA/Lip, (B) CUCA/Gal-Lip, (C) and CUCA/GA\&Gal-Lip. (D) The particle size changes of CUCA/GA\&Gal-Lip for $14 \mathrm{~d}$ in PBS and RPMI I640. (E) Drug release profiles of CUCA/GA\&Gal-Lip. Data expressed as mean \pm SD ( $\mathrm{n}=3$ ). 


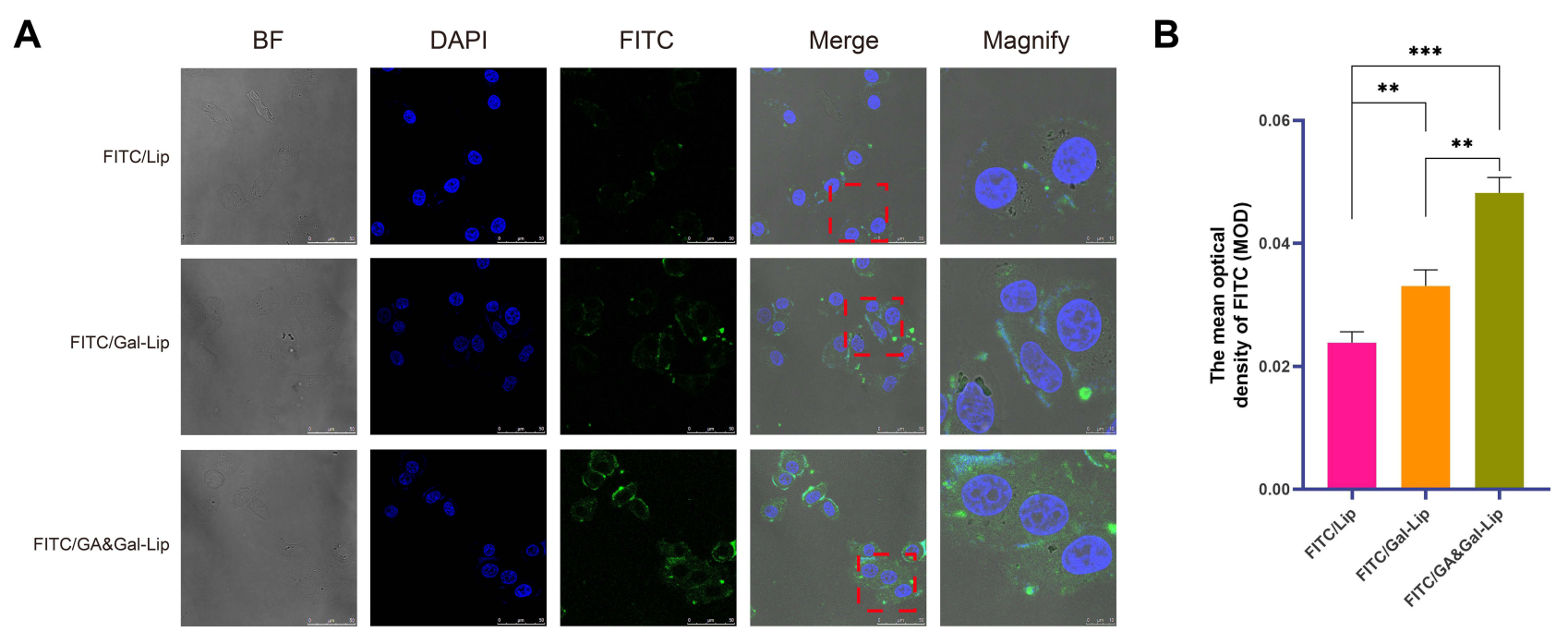

Figure 3 Cellular uptake of different liposomal formulations. (A) The cellular uptake of FITC-labeled Lip, FITC-labeled Gal-Lip and FITC-labeled GA\&Gal-Lip by BEL7402 cells for $0.5 \mathrm{~h}$. Green and blue fluorescence represented FITC and DAPI, respectively, and the magnified images were the areas of red dotted frames circle. (B) Quantitative histogram of the mean optical density of FITC of different Lip. Data expressed as mean $\pm S D(n=3)$. **P $<0.01$, ***P $<0.00$ I.

showed higher cytotoxicity compared to single-drug treatment (Figure 4F), suggesting that CUR and CA4P could exhibit synergistic anti-proliferative effects by proapoptotic and antiangiogenic activities in the co-cultured system.

\section{Cell Migration Assay}

To evaluate the anti-migration effects of different drug formulations, the cell migration assay was performed against BEL-7402 cells alone and BEL-7402/HUVEC co-cultured cells (Figure 5A). As shown in Figure 5B and $\mathrm{E}$, compared to free CUR or CA4P, BEL-7402 cells treated with CUR and CA4P combined groups showed larger wound areas, indicating greater anti-migration effects. In Figure 5C and $\mathrm{F}$, the HUVEC cells labeled by green fluorescence were located in the co-cultured system, and migrated into wounded area along with BEL-7402 cells after $24 \mathrm{~h}$. The migration rates in cocultured cells treated by CUCA/Gal-Lip and CUCA/ GA\&Gal-Lip were $12.4 \%$ and $7.3 \%$, respectively, which were lower than that of CUR/CA4P mixture (25.4\%) and CUCA/Lip (18.6\%). These results suggested that targeting Lip modified by Gal, or both of GA and Gal could have stronger inhibition ability on cell migration than non-targeting combination therapy. The possible explanation was that liver-targeting Lip significantly increased cellular internalization by GA/ Gal receptor-mediated endocytosis, resulting in greater anti-migration effect.

\section{Inhibitive Effect on VEGF Expression in vitro}

VEGF has been reported to play a crucial role in tumor angiogenesis, and $\mathrm{HCC}$ cells could promote proliferation of vascular endothelial cell by secreting VEGF. $^{42}$ Therefore, we evaluated the effects of different drug formulations on VEGF expression in BEL-7402/HUVEC cocultured cells through immunofluorescence assay. Figure $5 \mathrm{D}$ showed that the fluorescence intensity of CUR group was obviously reduced, indicating that CUR could effectively down-regulate the expression of VEGF. Compared to other combination formulations, CUCA/GA\&Gal-Lip displayed stronger inhibitory ability on VEGF expression (Figure 5G). This may be due to the fact that dual-ligandmodified Lip could improve the cellular internalization of drugs, leading to greater inhibition on VEGF expression.

\section{In vivo Biodistribution of Lip}

In order to determine liver-targeting properties of dual-ligand -modified Lip, the biological distribution of DiR-loaded liposomal formulation was observed by fluorescence imaging in vivo (Figure 6A). As shown in Figure 6B, the nearinfrared fluorescence imaging showed that the mice treated with two liposomal formulations had higher fluorescence in tumor region than free $\mathrm{DiR}$ group. The possible explanation is that nano-sized liposome improves DiR accumulation in tumor region by EPR effect. ${ }^{43}$ At $72 \mathrm{~h}$ post injection, DiR/ 
A

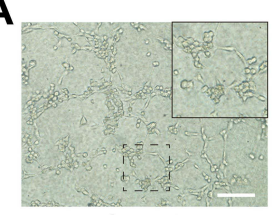

Control

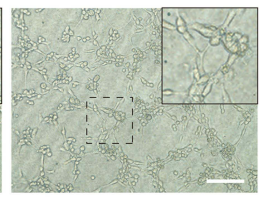

CUR

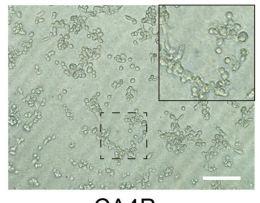

CA4P

$\mathbf{F}$

C

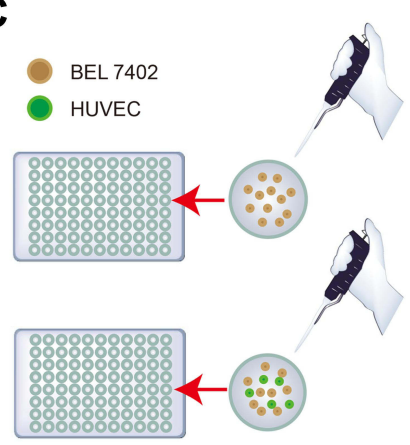

D

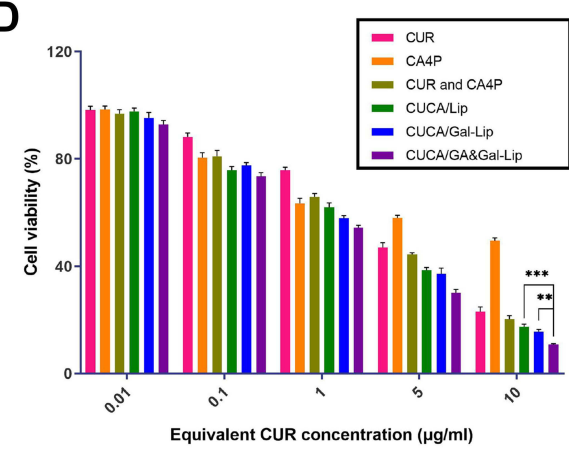

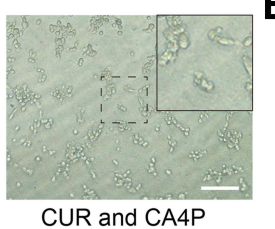

CUR and CA4P
B

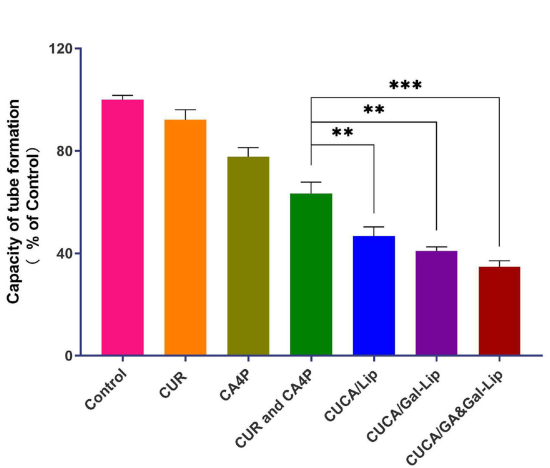

E

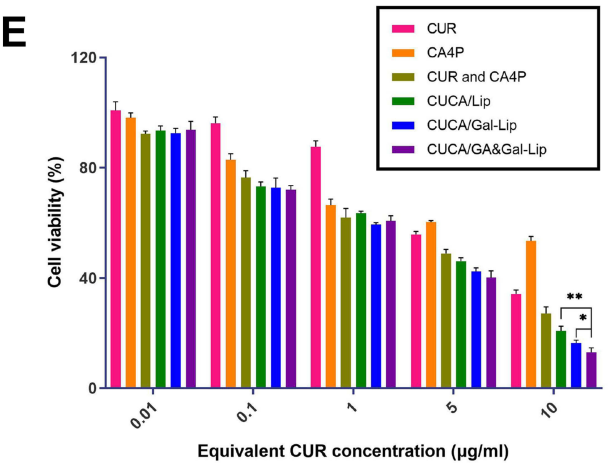

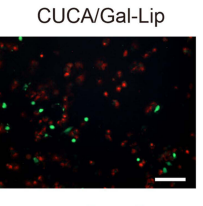

CUCA/GA\&Gal-Lip
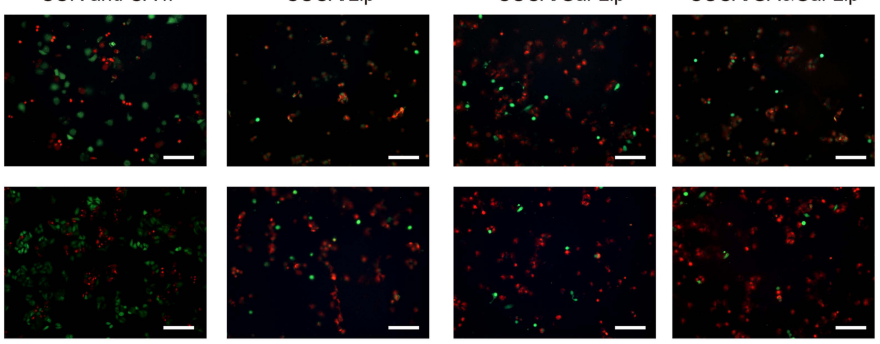

Figure 4 Effects of different Lip on HUVEC tube formation and viability of cells. (A) Typical images of tube formation and (B) HUVEC tube formation were quantified after treatment with various Lip groups. (C) The pattern diagram of BEL7402 cells alone and BEL7402/HUVEC co-cultured cells. Cytotoxicity of various Lip against (D) BEL7402 cells alone and (E) BEL7402/HUVEC co-cultured cells. (F) Typical images of Live/Dead staining of (A) alone BEL7402 cells and (B) co-cultured BEL7402 cells and HUVEC. Data expressed as mean \pm SD $(n=3)$. $* \mathrm{P}<0.05, * * \mathrm{P}<0.01$, *** $\mathrm{P}<0.001$. Scale bar, $500 \mu \mathrm{m}$.

Lip and DiR/GA\&Gal-Lip groups still exhibited strong fluorescence. Moreover, the mice injected with DiR/GA\&Gal-Lip group showed stronger fluorescence signal in the tumor region compared to DiR/Lip. The ex vivo imaging of tumors also showed that the fluorescence intensity of DiR/GA\&GalLip group was higher than that of DiR/Lip (Figure 6C and D). The results of in vivo distribution were consistent with those of in vitro cell uptake analysis.

\section{In vivo Antitumor Efficacy}

Tumor angiogenesis plays a crucial role in tumor development. To evaluate the anti-hepatoma effects of liver-targeting Lip co-loaded by proapoptotic and antiangiogenic drugs, H22 tumor-bearing mice were established and injected with different drug formulations (Figure 7A). Figure 7B shows that tumor growth in the drug treatment groups was suppressed in different degrees compared to saline group, and the tumor volumes in combinational groups were much smaller than that treated with CUR or CA4P alone, demonstrating that combination therapy with CUR and CA4P could improve the antitumor effects. Significantly, CUCA/ GA\&Gal-Lip showed higher tumor inhibition than nonligand/mono-ligand modified Lip with tumors gradually declining even after drug withdrawal, suggesting the dualligand-modified Lip could efficiently improve the antihepatoma efficacy by promoting the liver-targeting delivery of the drugs (Figure 7B and E). As illustrated in Figure 7D, CUCA/GA\&Gal-Lip exhibited superior anti-hepatoma 
A
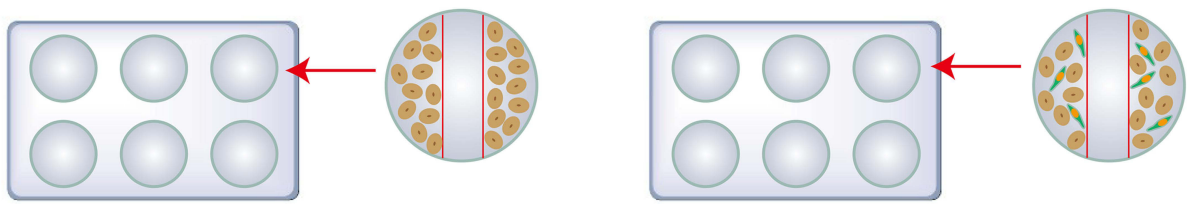

- HUVEC

BEL 7402
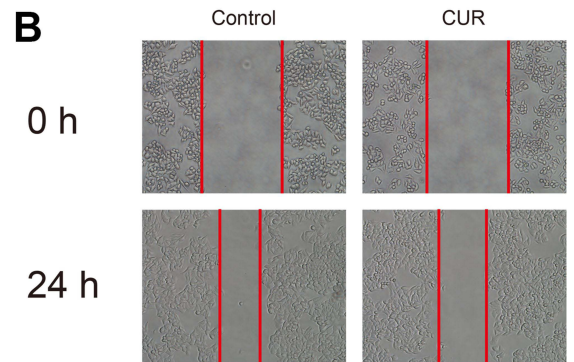

CA4P

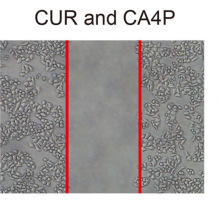

CUCA/Lip

CUCA/Gal-Lip

CUCA/GA\&Gal-Lip
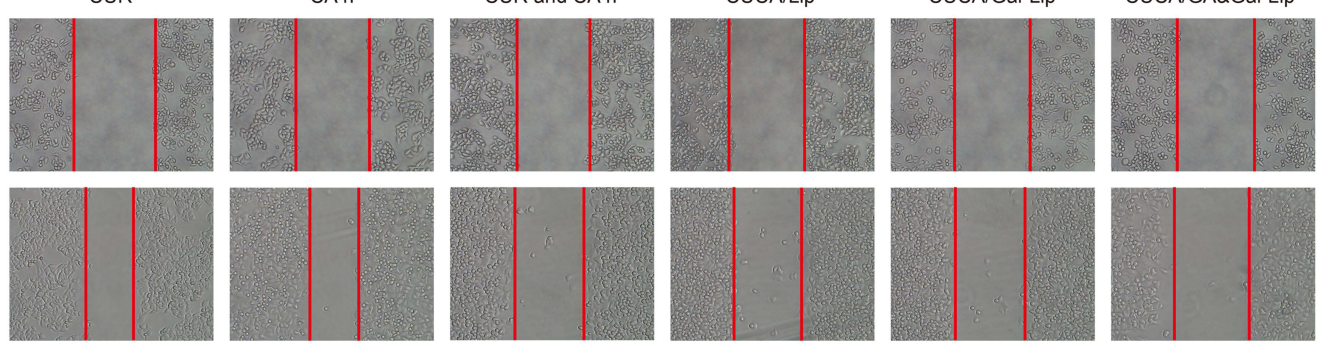

C
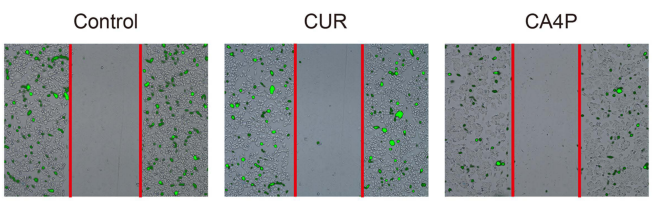

CUR and CA4P
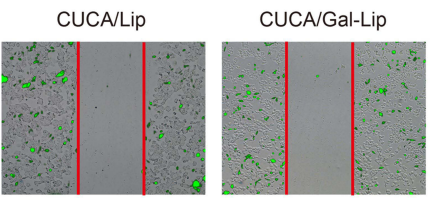

CUCA/GA\&Gal-Lip

$\mathrm{Oh}$

$24 \mathrm{~h}$
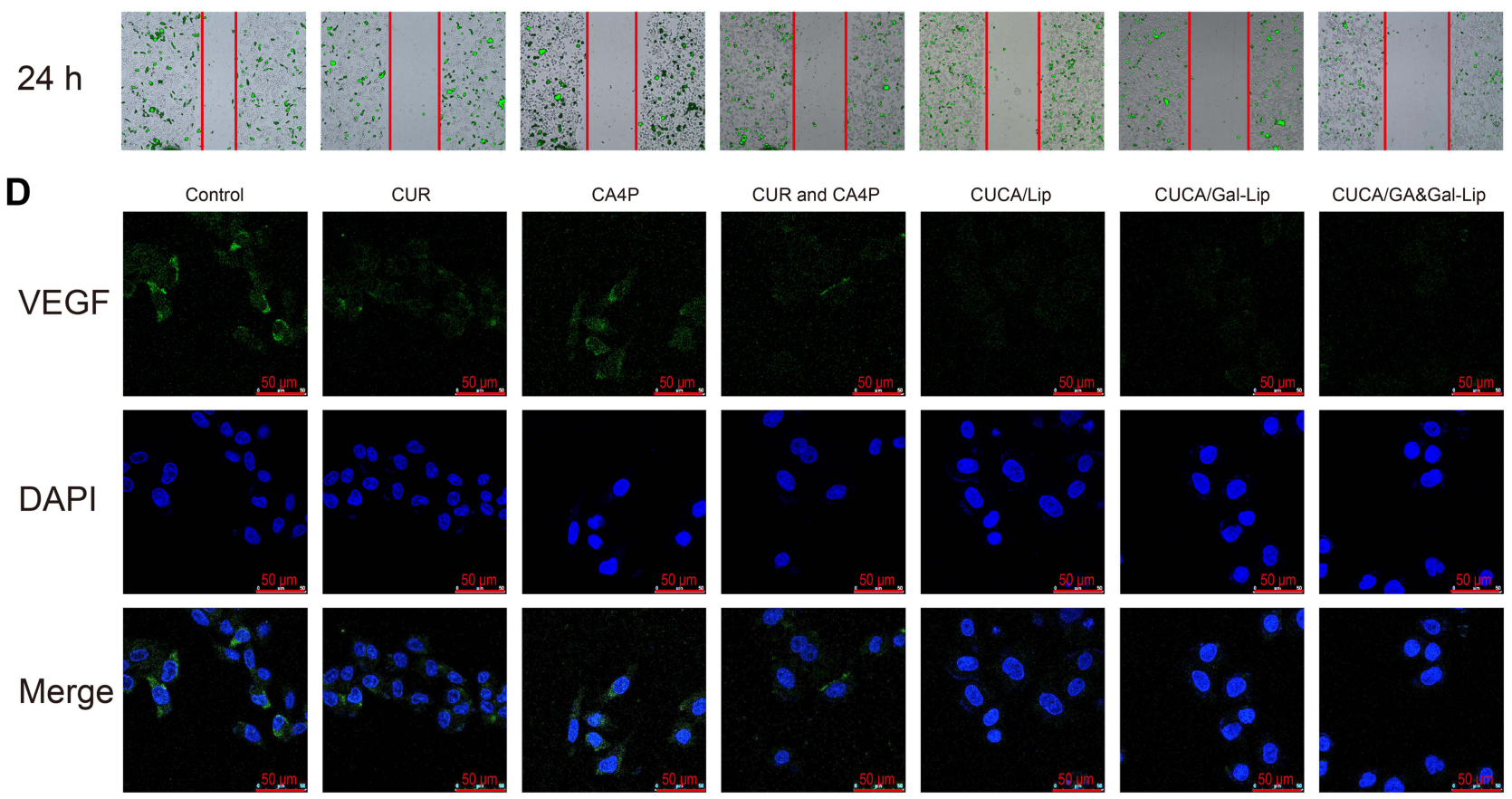

E

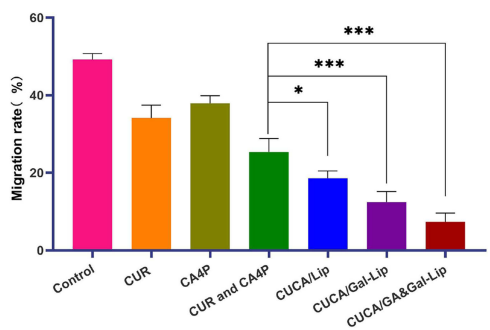

F

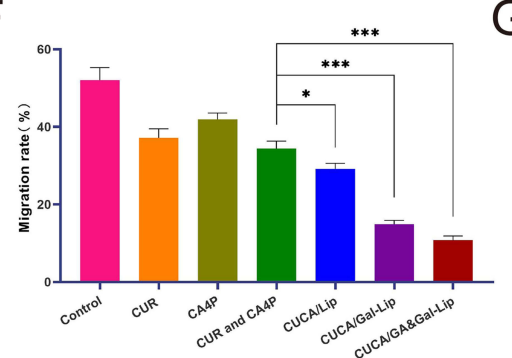

G

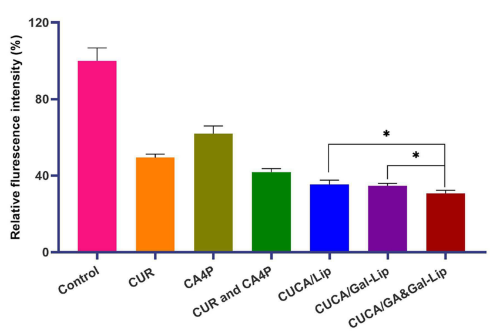

Figure 5 Effects of different Lip on cell migration and VEGF expression. (A) In vitro scratch experiment model of BEL7402 cells alone and BEL7402/HUVEC co-cultured cells. Typical images of different formulations inhibiting cell migration of (B) BEL7402 cells alone and (C) BEL7402/HUVEC co-cultured cells. (D) Immunofluorescence images of different drug formulations inhibiting VEGF expression. Green and blue colors represented VEGF and nuclei, respectively. The quantification of migration ability was presented as percentage of the wound area in (E) BEL7402 cells alone and (F) BEL7402/HUVEC co-cultured cells. (G) The quantification of inhibition of VEGF expression by different drug formulations. Data expressed as mean \pm SD $(n=3)$. *P $<0.05, * * * P<0.00 I$. 
A

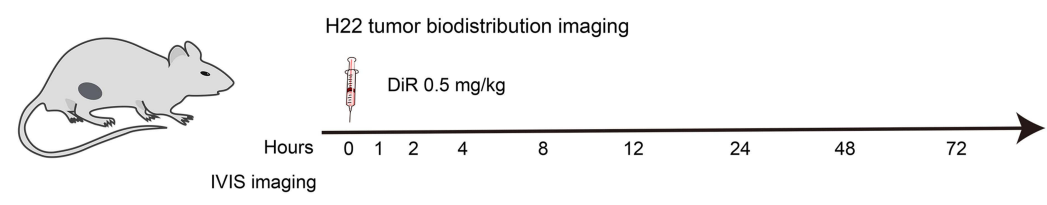

B

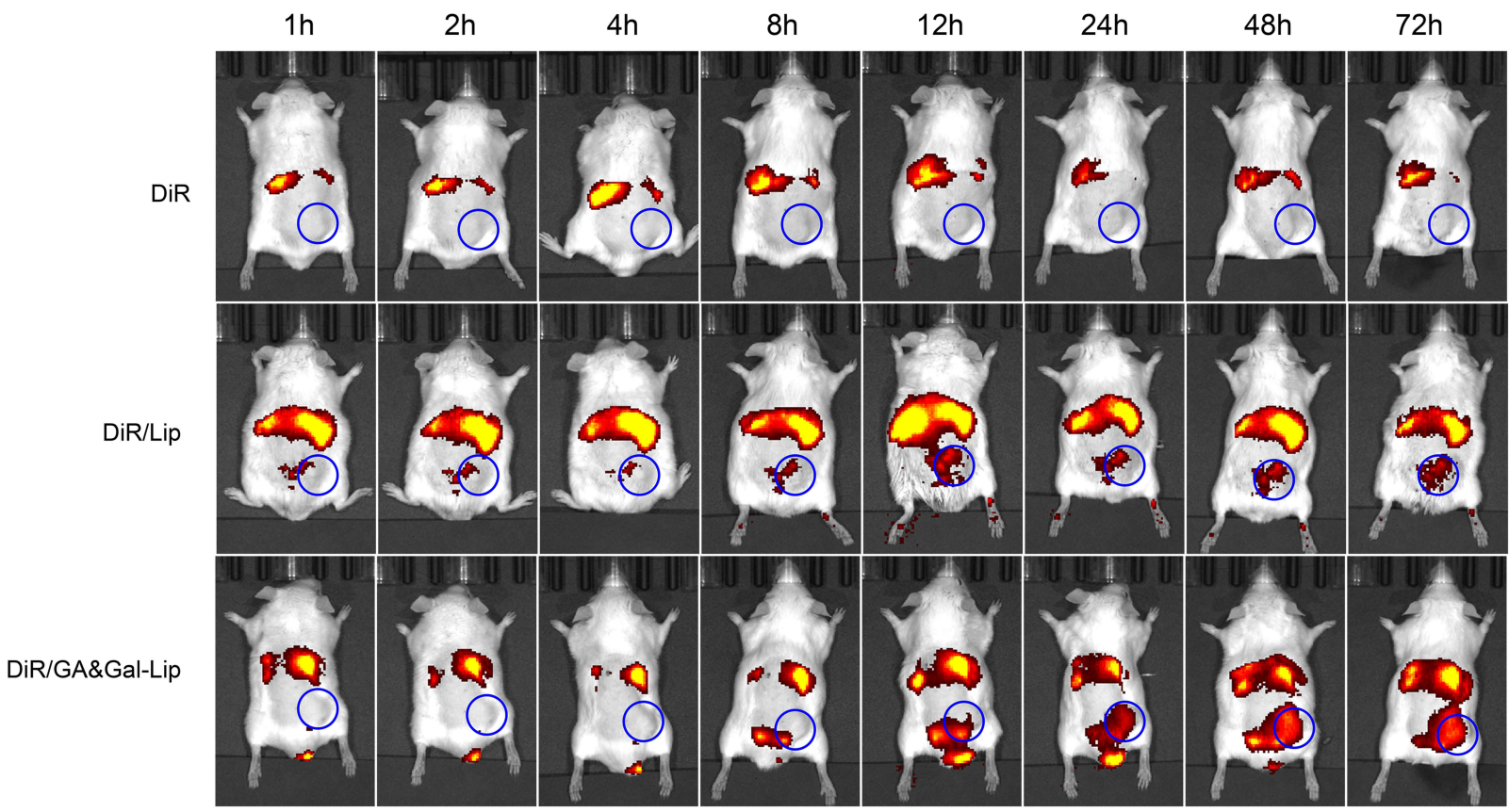

C

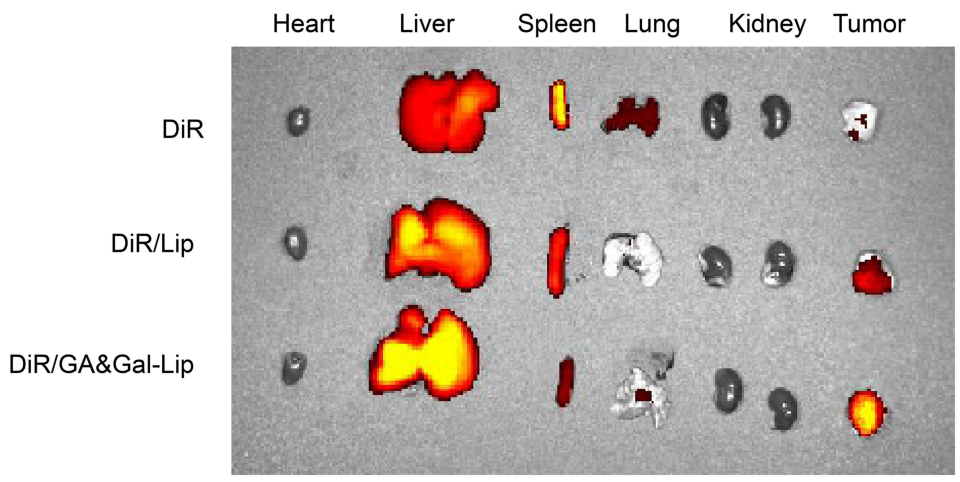

D

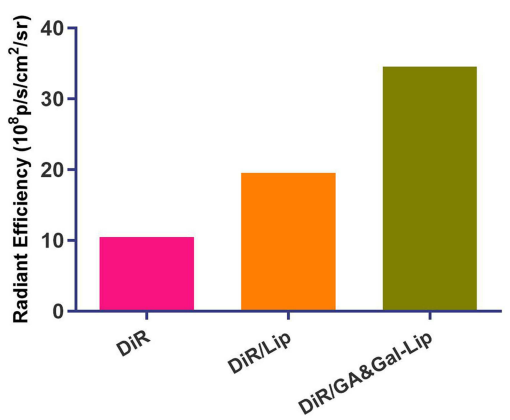

Figure 6 In vivo biodistribution of DiR, DiR/Lip and DiR/GA\&Gal-Lip. (A) Schematic design of in vivo biodistribution analysis. (B) IVIS images of H22 tumor-bearing BALB/c mice at different timepoints after administration of DiR, DiR/Lip and DiR/GA\&Gal-Lip. Blue circles represented the location of tumors. (C) Representative ex vivo IVIS images of organs and tumors. (D) Quantitative analysis for the tumors after $72 \mathrm{~h}$.

effects with the tumor inhibition rate reached to $93.5 \%$, which means the tumors were nearly declined.

In Figure 7C, it was shown that the weights of the mice treated with liposomal formulations have no significant changes compared to the control group, indicating that the Lip were safe nano-carriers for drug delivery. After treatment, the organs and tumor of H22 tumor-bearing mice were excised for $H \& E$ staining analysis. As shown in Supporting Figure 2, vacuolation and dissolution of myocardial fibers in heart occurred in all free drug groups, indicating that free CUR, free CA4P or the mixture of two free drugs had some degree of cardiotoxicity. By contrast, there was no visualized degeneration of myocardial fibers in all liposomal groups. Meanwhile, no significant difference was observed in liver, spleen, lung and kidney in three liposomal groups compared to the saline group. All the above results revealed that the combination therapy based on Lip had no significant systemic toxicity.

In addition, the $H \& E$ staining images of tumors are listed in Figure 7F. The tumor region in all drug treatment groups 
A

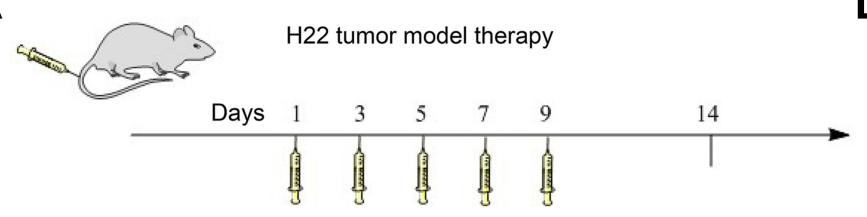

B

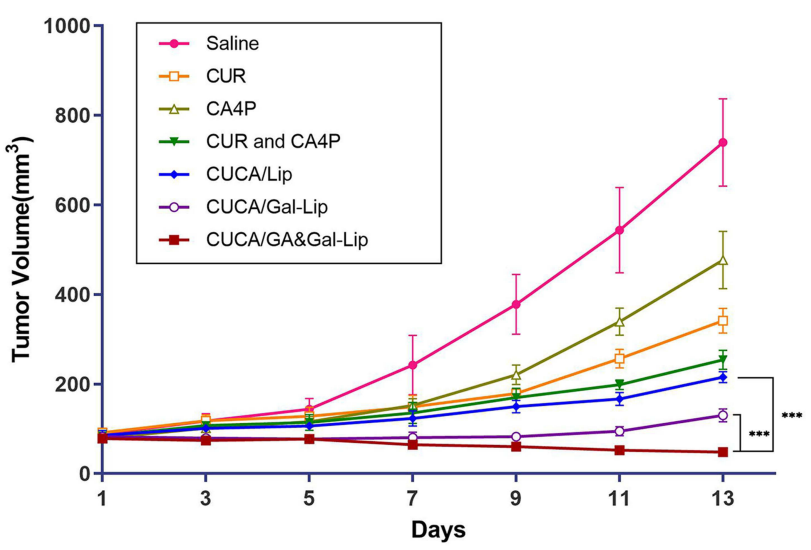

C

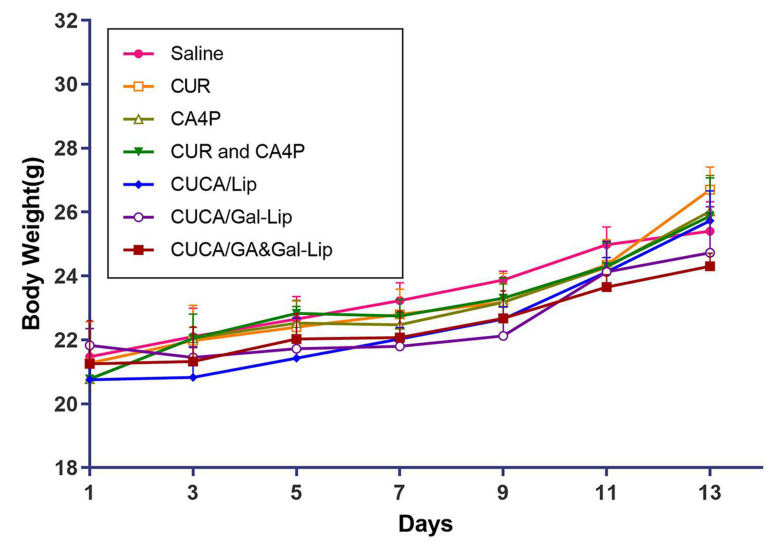

D

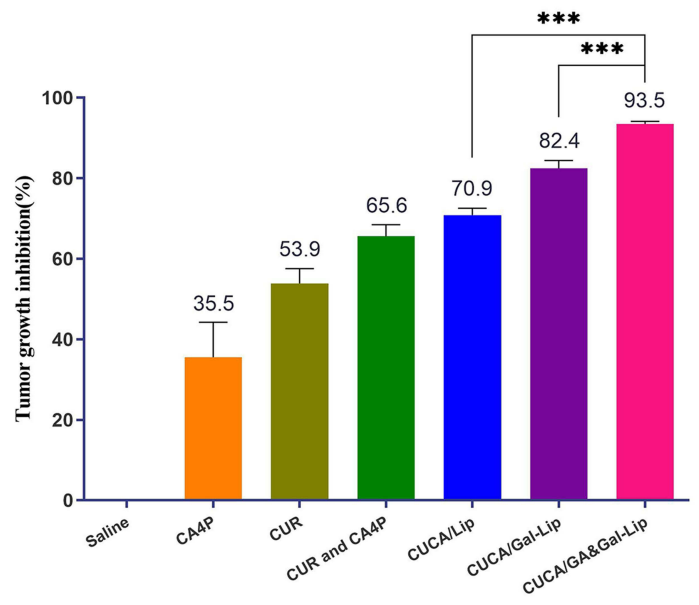

E

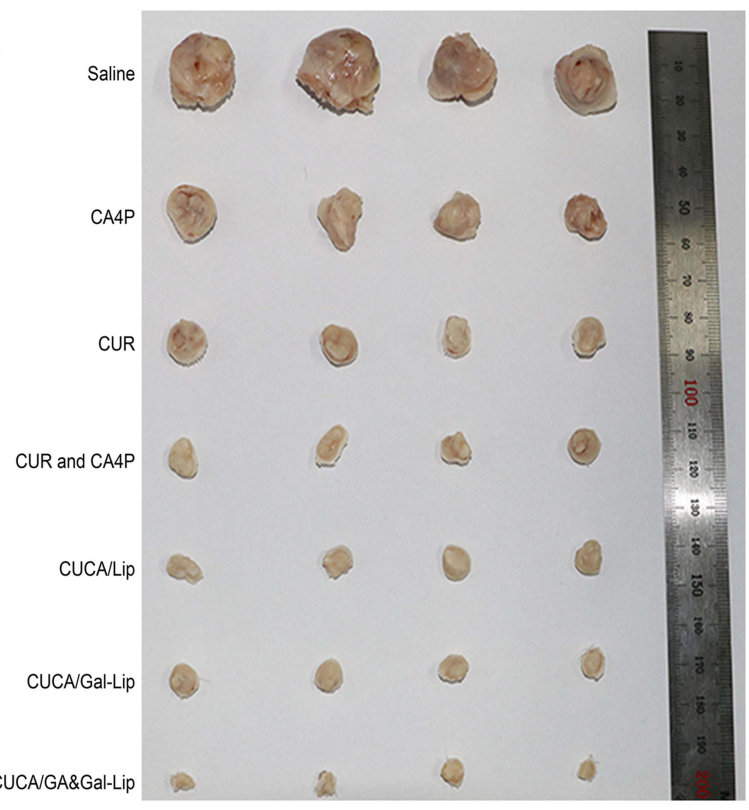

F

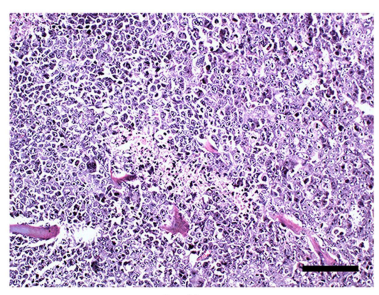

Saline

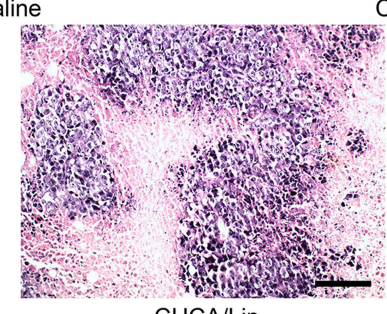

CUCA/Lip

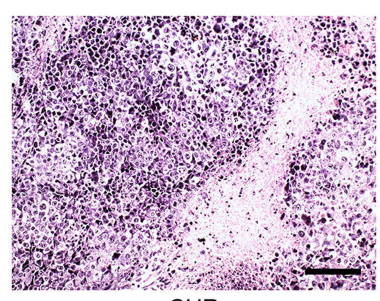

CUR

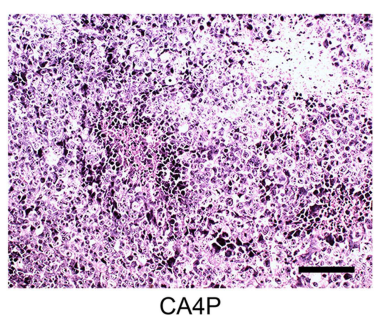

CA4P

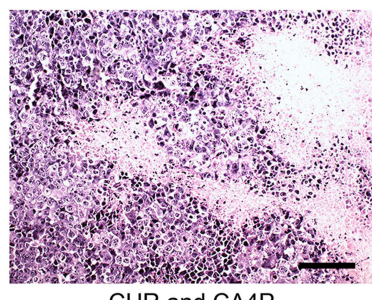

CUR and CA4P

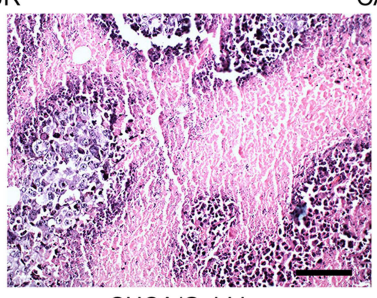

CUCA/Gal-Lip

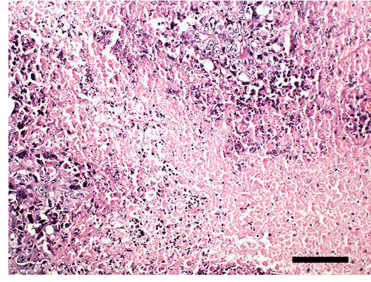

CUCA/GA\&Gal-Lip

Figure 7 In vivo antitumor efficacy of CUCA/GA\&Gal-Lip. (A) Schematic design of antitumor therapy. (B) the tumor volumes and (C) body weights were measured once every other day for 7 times. (D) The tumor growth-inhibition rates and (E) images of excised tumors of different formulations treatment groups. (F) Images of H\&E staining for tumors. ${ }^{* * * P}<0.001$. Data expressed as mean $\pm S D(n=4)$. Scale bar, $100 \mu \mathrm{m}$. 
exhibited obvious morphologic changes, with obvious karyolysis and cytoplasmic vacuolation. More importantly, the necrotic degree of the CUCA/GA\&Gal-Lip group was greater than that of the other groups, showing that CUCA/ GA\&Gal-Lip had superior antitumor effects.

\section{Immunohistochemistry Analysis}

Blood vessels play a crucial role in tumor growth and metastasis. In order to evaluate the in vivo anti-angiogenic effects of combination therapy, the microvascular density (MVD), VEGF and VEGFR2 expression were investigated by immunohistochemistry analysis. CD31, also known as platelet-endothelial cell adhesion molecule, is a commonly used endothelial marker. Therefore, CD31 was selected to evaluate tumor angiogenesis. As shown in Figure 8A and B, the brown microvessels stained were easily visualized in the control saline group, while the number of microvessels in CUR or CAP group obviously reduced, indicating that both drugs could inhibit tumor angiogenesis. Significantly, CUCA/GA\&Gal-Lip exhibited stronger anti-angiogenic effects than other liposomal formulations, suggesting that dual-ligand-modified Lip could efficiently inhibit tumor angiogenesis in tumor microenvironment.

To explore the anti-angiogenic mechanism of combination therapy, the VEGF and VEGFR2 expression were further analyzed in vivo. Figure 8C shows that CUR significantly reduced the expression of VEGF, while CA4P exerted little anti-VEGF effects, suggesting that CUR inhibited tumorangiogenesis by down-regulating the VEGF expression. ${ }^{15}$ In contrast, the CA4P exhibited stronger inhibitory effects on VEGFR2 expression than CUR, indicating that CA4P achieved anti-angiogenesis by reducing the VEGFR2 level in vascular endothelial cells. ${ }^{10}$ The results revealed that CUR and CA4P could inhibit tumor angiogenesis by the mechanism of anti-VEGF and anti-VEGFR2, respectively, and that the combination therapy of CUR and CA4P could more significantly inhibit tumor angiogenesis by simultaneously blocking the VEGF/VEGFR2 signaling pathway.

\section{Dual-Ligand-Modified Lip Inhibits Lung Metastasis}

Extrahepatic metastasis is one of the main causes of poor prognosis of HCC, and tumor angiogenesis was crucial for tumor invasion and metastasis. Lung, lymph nodes and bones are the usual region of extrahepatic metastasis from HCC, among which lung showed the highest rate of metastasis. ${ }^{44}$ To evaluate the anti-metastatic effects of different drug formulations, we established lung metastasis model by injecting $\mathrm{H} 22$ cells into tail vein of mice (Figure 9A). As shown in the Figure 9B and D, compared to saline control group, the numbers of pulmonary nodules treated by free CUR and free CA4P was
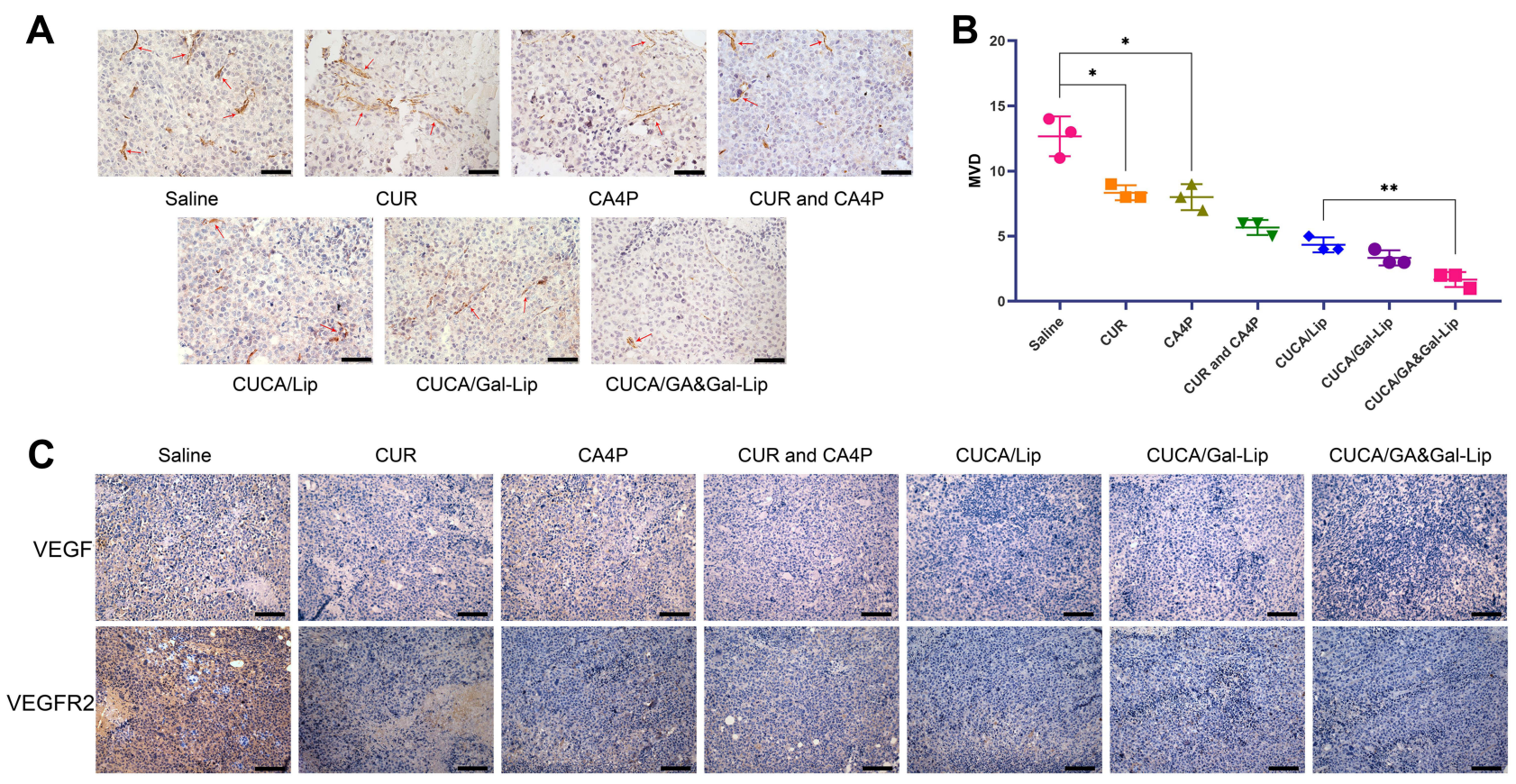

Figure 8 Images of immunohistochemistry analysis. (A) Representative images of tumor sections stained with CD3I. Red arrowheads indicate the tumor microvessels. (B) Quantification of microvessel density (MVD). (C) Immunohistochemical staining of VEGF and VEGFR2 in tumor tissues. Data expressed as mean \pm SD ( $\mathrm{n}=3$ ). *P $<0.05$, **P $<0.01$. Scale bar, $100 \mu \mathrm{m}$. 


\section{A}

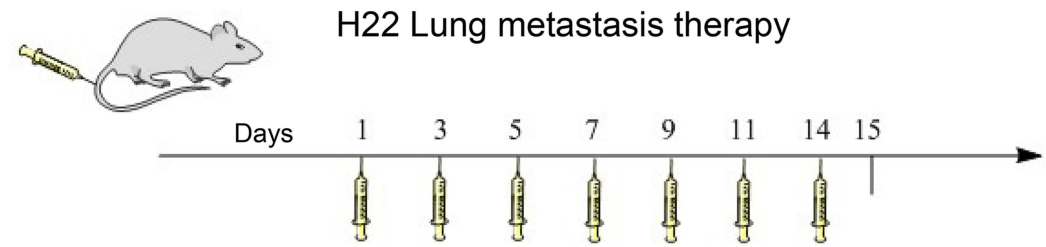

B

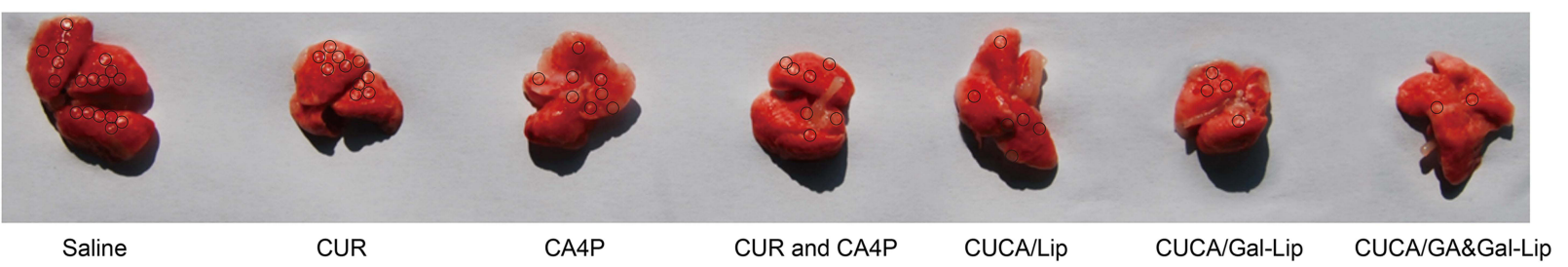

C

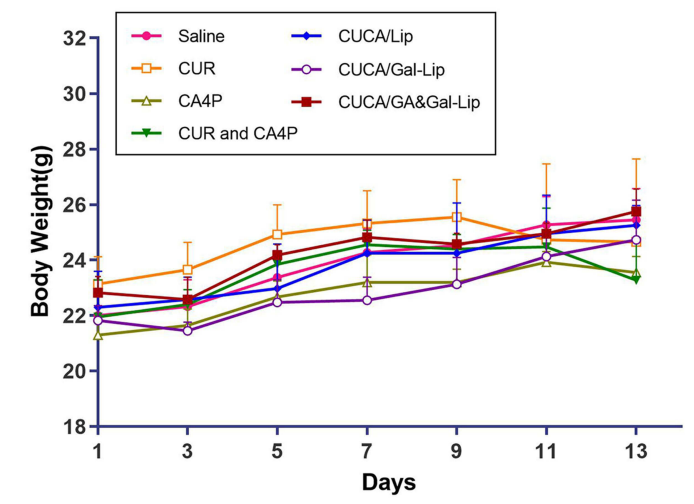

D

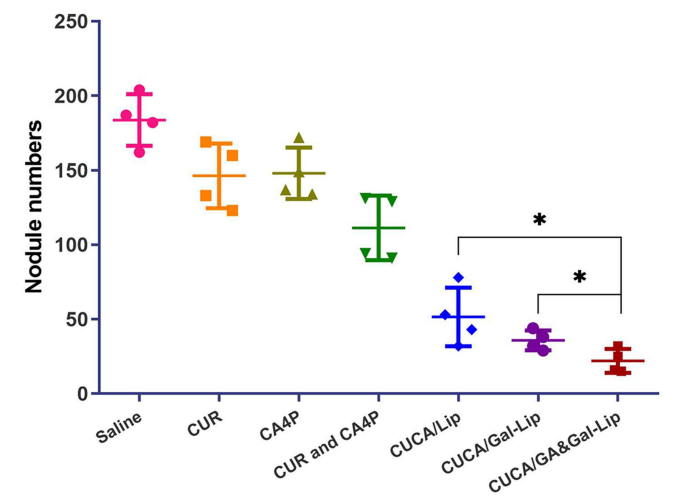

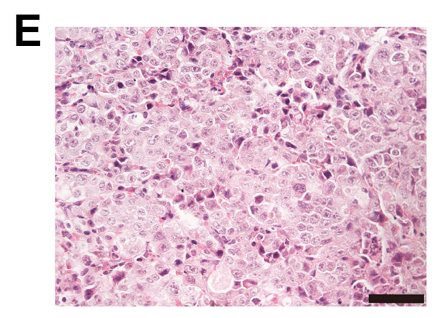

Saline

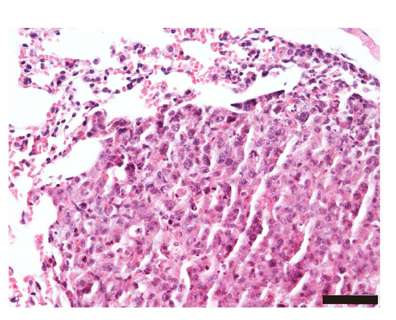

CUR

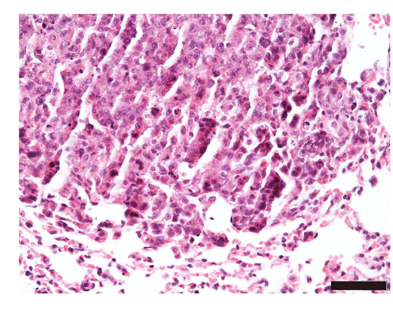

CA4P

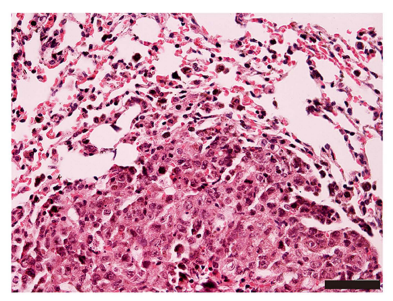

CUR and CA4P

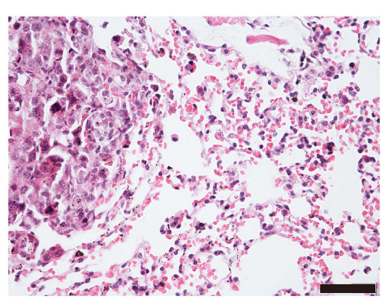

CUCA/Lip

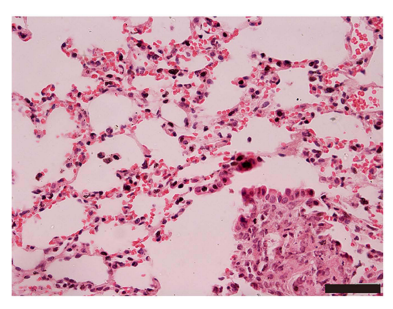

CUCA/Gal-Lip

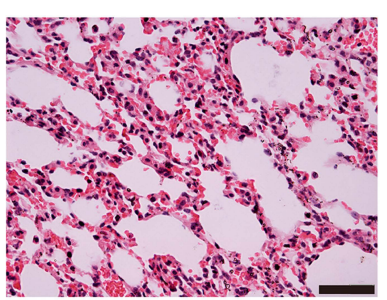

CUCA/GA\&Gal-Lip

Figure 9 Effects of different formulations on lung metastasis in vivo. (A) Schematic design of anti-metastatic therapy. (B) Morphologies of lungs excised in different treatment groups. (C) The changes in body weight of different treatment groups. (D) Quantification of metastasis node numbers. (E) Images of H\&E staining for lungs. Data expressed as mean $\pm S D(n=4)$. $* P<0.05$. Scale bar, $100 \mu m$.

slightly reduced, indicating that single drug exerted weak inhibitory effects on lung metastasis. As expected, the nodule number observably decreased in the co-delivery Lip groups. The results revealed that combination therapy basing on nanosized Lip exhibited strong anti-metastatic effects. Moreover, the mice treated with CUCA/GA\&Gal-Lip showed less lung metastasis compared to other liposomal formulations. This is due to the fact that the GA receptor and ASGP-R are overexpressed on the cytomembrane of $\mathrm{H} 22$ cells, and the dualligand-modified Lip could promote drug accumulation in the lung metastatic foci by active-targeting delivery, leading to superior anti-metastatic effects. As illustrated in Figure 9C, 
the mice treated with all liposomal groups had no significant changes in body weight in comparison with saline control group. Moreover, the morphometrics (Figure 9B) and H\&E staining images (Figure 9E) of lung organ showed that there were almost no lung metastasis foci in the mice treated with CUCA/GA\&Gal-Lip, suggesting that the combination therapy based on dual-ligand modified Lip had great potentials for efficient inhibition of HCC growth and metastasis.

\section{Conclusion}

In this study, novel dual-ligand-modified Lip co-loaded with CUR and CA4P were firstly developed for antihepatoma therapy. Compared to other liposomal groups, CUCA/GA\&GA-Lip exhibited superior inhibitory effects on cellular proliferation and migration against BEL-7402 and BEL-7402/HUVEC co-cultured cells, and significantly inhibited tube formation in vitro. The in vivo antitumor study showed that CUCA/GA\&GA-Lip could more effectively inhibit tumor angiogenesis and metastasis by simultaneously blocking VEGF/VEGFR2 signal pathway, and improved anti-hepatoma efficacy through introducing GA and Gal ligands onto the Lip. Summarily, GA/Galmodified Lip co-loaded of CUR and CA4P are a promising approach for anti-angiogenic and antimetastatic therapy in anti-hepatoma treatment with great application prospects.

\section{Acknowledgments}

This work was supported by the National Science Foundation of China (81803464), Natural Science Foundation of Shandong Province (ZR2018BH041, ZR2020MC078), the Education Science and Technology Project of Shandong Province (J17KA141).

\section{Disclosure}

The authors report no conflicts of interest in this work.

\section{References}

1. Anwanwan D, Singh SK, Singh S, et al. Challenges in liver cancer and possible treatment approaches. Biochim Biophys Acta Rev Cancer. 2020;1873(1):188314. doi:10.1016/j.bbcan.2019.188314

2. Bray F, Ferlay J, Soerjomataram I, et al. Global cancer statistics 2018: GLOBOCAN estimates of incidence and mortality worldwide for 36 cancers in 185 countries. CA Cancer J Clin. 2018;68(6):394-424. doi:10.3322/caac. 21492

3. Perez-Herrero E. Fernandez-Medarde A Advanced targeted therapies in cancer: drug nanocarriers, the future of chemotherapy. Eur J Pharm Biopharm. 2015;93:52-79. doi:10.1016/j.ejpb.2015.03.018
4. Zhu D, Wu S, Hu C, et al. Folate-targeted polymersomes loaded with both paclitaxel and doxorubicin for the combination chemotherapy of hepatocellular carcinoma. Acta Biomater. 2017;58:399-412. doi:10.1016/j.actbio.2017.06.017

5. Li F, Wang Y, Chen WL, et al. Co-delivery of VEGF siRNA and etoposide for enhanced anti-angiogenesis and anti-proliferation effect via multi-functional nanoparticles for orthotopic non-small cell lung cancer treatment. Theranostics. 2019;9(20):5886-5898. doi:10.7150/ thno. 32416

6. Abdalla AME, Xiao L, Ullah MW, et al. Current challenges of cancer anti-angiogenic therapy and the promise of nanotherapeutics. Theranostics. 2018;8(2):533-548. doi:10.7150/thno.21674

7. Heinolainen K, Karaman S, D'Amico G, et al. VEGFR3 modulates vascular permeability by controlling VEGF/VEGFR2 signaling. Circ Res. 2017;120(9):1414-1425. doi:10.1161/CIRCRESAHA.116. 310477

8. Van der Veldt AA, Lubberink M, Bahce I, et al. Rapid decrease in delivery of chemotherapy to tumors after anti-VEGF therapy: implications for scheduling of anti-angiogenic drugs. Cancer Cell. 2012;21 (1):82-91. doi:10.1016/j.ccr.2011.11.023

9. Abdollahi A. Folkman J Evading tumor evasion: current concepts and perspectives of anti-angiogenic cancer therapy. Drug Resist Updat. 2010;13(1-2):16-28. doi:10.1016/j.drup.2009.12.001

10. Su M, Huang J, Liu S, et al. The anti-angiogenic effect and novel mechanisms of action of Combretastatin A-4. Sci Rep. 2016;6:28139. doi:10.1038/srep28139

11. Uddin S, Hussain AR, Manogaran PS, et al. Curcumin suppresses growth and induces apoptosis in primary effusion lymphoma. Oncogene. 2005;24(47):7022-7030. doi:10.1038/sj.onc.1208864

12. Hussain AR, Al-Rasheed M, Manogaran PS, et al. Curcumin induces apoptosis via inhibition of PI3'-kinase/AKT pathway in acute T cell leukemias. Apoptosis. 2006;11(2):245-254. doi:10.1007/s10495-006-3392-3

13. Bill MA, Fuchs JR, Li C, et al. The small molecule curcumin analog FLLL32 induces apoptosis in melanoma cells via STAT3 inhibition and retains the cellular response to cytokines with antitumor activity. Mol Cancer. 2010;9:165. doi:10.1186/1476-4598-9-165

14. Freudlsperger C, Greten J. Schumacher U Curcumin induces apoptosis in human neuroblastoma cells via inhibition of NFkappaB. Anticancer Res. 2008;28(1A):209-214.

15. Shan B, Schaaf C, Schmidt A, et al. Curcumin suppresses HIF1A synthesis and VEGFA release in pituitary adenomas. $J$ Endocrinol. 2012;214(3):389-398. doi:10.1530/JOE-12-0207

16. Mohan R, Sivak J, Ashton P, et al. Curcuminoids inhibit the angiogenic response stimulated by fibroblast growth factor-2, including expression of matrix metalloproteinase gelatinase B. J Biol Chem. 2000;275(14):10405-10412. doi:10.1074/jbc.275.14.10405

17. Cho K, Wang $\mathrm{X}$, Nie S, et al. Therapeutic nanoparticles for drug delivery in cancer. Clin Cancer Res. 2008;14(5):1310-1316. doi:10.1158/1078-0432.CCR-07-1441

18. Riaz MK, Riaz MA, Zhang X, et al. Surface Functionalization and Targeting Strategies of Liposomes in Solid Tumor Therapy: a Review. Int J Mol Sci. 2018;19:154. doi:10.3390/ijms19010195

19. Abdelmoneem MA, Elnaggar MA, Hammady RS, et al. Dual-Targeted Lactoferrin Shell-Oily Core Nanocapsules for Synergistic Targeted/ Herbal Therapy of Hepatocellular Carcinoma. ACS Appl Mater Interfaces. 2019;11(30):26731-26744. doi:10.1021/acsami.9b10164

20. Tian Q, Zhang CN, Wang XH, et al. Glycyrrhetinic acid-modified chitosan/poly(ethylene glycol) nanoparticles for liver-targeted delivery. Biomaterials. 2010;31(17):4748-4756. doi:10.1016/j. biomaterials.2010.02.042

21. Sun Y, Dai C, Yin M, et al. Hepatocellular carcinoma-targeted effect of configurations and groups of glycyrrhetinic acid by evaluation of its derivative-modified liposomes. Int $J$ Nanomedicine. 2018;13:1621-1632. doi:10.2147/IJN.S153944 
22. Guo P, Yang J, Liu D, et al. Dual complementary liposomes inhibit triple-negative breast tumor progression and metastasis. Sci Adv. 2019;5(3):eaav5010. doi:10.1126/sciadv.aav5010

23. Stefanick JF, Omstead DT, Kiziltepe T, et al. Dual-receptor targeted strategy in nanoparticle design achieves tumor cell selectivity through cooperativity. Nanoscale. 2019;11(10):4414-4427. doi:10.1039/ c8nr09431d

24. Li X, Zhou H, Yang L, et al. Enhancement of cell recognition in vitro by dual-ligand cancer targeting gold nanoparticles. Biomaterials. 2011;32(10):2540-2545. doi:10.1016/j.biomaterials.2010.12.031

25. Li Z, Zheng Z, Li C, et al. Therapeutic drugs and drug delivery systems targeting stromal cells for cancer therapy: a review. J Drug Target. 2020;28(7-8):714-726. doi:10.1080/ 1061186X.2020.1744157

26. Quail DF, Joyce JA. Joyce J A Microenvironmental regulation of tumor progression and metastasis. Nat Med. 2013;19(11):1423-1437. doi: $10.1038 / \mathrm{nm} .3394$

27. Chen X, Song E. Song E Turning foes to friends: targeting cancer-associated fibroblasts. Nat Rev Drug Discov. 2019;18 (2):99-115. doi:10.1038/s41573-018-0004-1

28. Gajewski TF, Schreiber H, Fu YX. Innate and adaptive immune cells in the tumor microenvironment. Nat Immunol. 2013;14 (10):1014-1022. doi:10.1038/ni.2703

29. Hida K, Hida Y. Shindoh M Understanding tumor endothelial cell abnormalities to develop ideal anti-angiogenic therapies. Cancer Sci. 2008;99(3):459-466. doi:10.1111/j.1349-7006.2007.00704.x

30. Jiang H, Li ZP, Tian GX, et al. Liver-targeted liposomes for codelivery of curcumin and combretastatin A4 phosphate: preparation, characterization, and antitumor effects. Int $J$ Nanomedicine. 2019;14:1789-1804. doi:10.2147/IJN.S188971

31. Qi C, Wang D, Gong X, et al. Co-delivery of curcumin and capsaicin by dual-targeting liposomes for inhibition of aHSC-induced drug resistance and metastasis. ACS Appl Mater Interfaces. 2021;13 (14):16019-16035. doi:10.1021/acsami.0c23137

32. Li C, Guan H, Li Z, et al. Study on different particle sizes of DOX-loaded mixed micelles for cancer therapy. Colloids Surf B Biointerfaces. 2020;196:111303. doi:10.1016/j.colsurfb.2020.111303

33. Park HA, Licznerski P, Mnatsakanyan N, et al. Inhibition of Bcl-xL prevents pro-death actions of DeltaN-Bcl-xL at the mitochondrial inner membrane during glutamate excitotoxicity. Cell Death Differ. 2017;24(11):1963-1974. doi:10.1038/cdd.2017.123

34. Chen CY, Rao SS, Ren L, et al. Exosomal DMBT1 from human urine-derived stem cells facilitates diabetic wound repair by promoting angiogenesis. Theranostics. 2018;8(6):1607-1623. doi:10.7150/ thno. 22958
35. Wu H, Luo Y, Xu D, et al. Low molecular weight heparin modified bone targeting liposomes for orthotopic osteosarcoma and breast cancer bone metastatic tumors. Int $J$ Biol Macromol. 2020;164:2583-2597. doi:10.1016/j.ijbiomac.2020.08.068

36. Li ZP, Tian GX, Jiang $\mathrm{H}$, et al. Liver-targeting and $\mathrm{pH}$-sensitive sulfated hyaluronic acid mixed micelles for hepatoma therapy. Int J Nanomedicine. 2019;14:9437-9452. doi:10.2147/IJN.S214528

37. Yue W, Chen L, Yu L, et al. Checkpoint blockade and nanosonosensitizer-augmented noninvasive sonodynamic therapy combination reduces tumour growth and metastases in mice. Nat Commun. 2019;10(1):2025. doi:10.1038/s41467-019-09760-3

38. Xu J, Cheng X, Tan L, et al. Microwave rsponsive nanoplatform via P-selectin mediated drug delivery for treatment of hepatocellular carcinoma with distant metastasis. Nano Lett. 2019;19 (5):2914-2927. doi:10.1021/acs.nanolett.8b05202

39. Hu T, Cao H, Yang C, et al. LHD-modified mechanism-based liposome coencapsulation of mitoxantrone and prednisolone using novel lipid bilayer fusion for tissue-specific colocalization and synergistic antitumor effects. ACS Appl Mater Interfaces. 2016;8 (10):6586-6601. doi:10.1021/acsami.5b10598

40. Liu X, Han M, Xu J, et al. Asialoglycoprotein receptor-targeted liposomes loaded with a norcantharimide derivative for hepatocyte-selective targeting. Int J Pharm. 2017;520(1-2):98-110. doi:10.1016/j.ijpharm.2017.02.010

41. Cai Y, Xu Y, Chan HF, et al. Glycyrrhetinic acid mediated drug delivery carriers for hepatocellular carcinoma therapy. Mol Pharm. 2016;13(3):699-709. doi:10.1021/acs.molpharmaceut.5b00677

42. Song F, Chen Q, Rao W, et al. OVA66 promotes tumour angiogenesis and progression through enhancing autocrine VEGF-VEGFR2 signalling. EBioMedicine. 2019;41:156-166. doi:10.1016/j. ebiom.2019.02.051

43. Fang J, Islam W, Maeda H. Maeda H Exploiting the dynamics of the EPR effect and strategies to improve the therapeutic effects of nanomedicines by using EPR effect enhancers. Adv Drug Deliv Rev. 2020;157:142-160. doi:10.1016/j.addr.2020.06.005

44. Uka K, Aikata H, Takaki S, et al. Clinical features and prognosis of patients with extrahepatic metastases from hepatocellular carcinoma. World J Gastroenterol. 2007;13(3):414-420. doi:10.3748/wjg.v13. i3.414
International Journal of Nanomedicine

\section{Publish your work in this journal}

The International Journal of Nanomedicine is an international, peerreviewed journal focusing on the application of nanotechnology in diagnostics, therapeutics, and drug delivery systems throughout the biomedical field. This journal is indexed on PubMed Central, MedLine, CAS, SciSearch ${ }^{\mathbb{R}}$, Current Contents ${ }^{\mathbb{R}} /$ Clinical Medicine, $^{2}$

\section{Dovepress}

Journal Citation Reports/Science Edition, EMBase, Scopus and the Elsevier Bibliographic databases. The manuscript management system is completely online and includes a very quick and fair peer-review system, which is all easy to use. Visit http://www.dovepress.com/ testimonials.php to read real quotes from published authors. 